\title{
Sahlqvist correspondence for modal mu-calculus
}

\author{
Johan van Benthem, Nick Bezhanishvili, Ian Hodkinson
}

August 2, 2011

\begin{abstract}
We define analogues of modal Sahlqvist formulas for the modal mucalculus, and prove a correspondence theorem for them.
\end{abstract}

\section{Introduction}

The modal mu-calculus provides a perspicuous way of isolating essential laws of induction and recursion generalizing computational logics such as PDL, CTL, and CTL*. This paper adds one more strand to its exploration, going back to a traditional modal concern: frame correspondence theory. It was observed in [5] how the usual method for obtaining frame correspondents for Sahlqvisttype axioms can be applied to non-first-order axioms like Löb's Axiom whose antecedents have a special 'PIA syntax' supporting a minimal valuation that is definable in the classical fixed-point language FO+LFP. It is then natural to look for a balance on both sides, in terms of generalized Sahlqvist forms in the language of the modal mu-calculus that support this style of analysis. Such a generalization is found in this paper, by employing additional notions and techniques from [6]. We will use only semantic standard models here, but the latter paper also considers generalized models for the mu-calculus with restrictions on the predicates that are available in the process of fixed-point approximation.

We will not look into completeness versions of Sahlqvist's Theorem in this paper, except for a few remarks on the existence of proof systems that match semantic frame correspondence arguments. However, this research is part of a larger project on analyzing special-purpose logics based on the modal mucalculus, and finding general techniques for their completeness proofs, which are still lacking today. An important bridge in obtaining completeness from correspondence results for Sahlqvist axioms has been the celebrated Esakia Lemma [12] tying modal semantics to topological spaces. This is just one of the many strategic points in research on modal logic and beyond where Leo Esakia has shown the way to so many of us. We are happy to dedicate this article to the memory of this great teacher, colleague, and friend. 


\section{Preliminaries}

Before we start, we briefly go through the background material and notation needed for the paper. Our terseness is due to lack of space.

\section{$2.1 \quad$ Modal mu-calculus}

We fix disjoint sets $\mathcal{P}$ of propositional atoms and $\mathcal{V}$ of fixed point variables. We write $p, q, s, \ldots$ for propositional atoms, and $X, Y, Z, \ldots$ for fixed point variables.

Any element of $\mathcal{P} \cup \mathcal{V}$ is a modal mu-formula, as are $T, \perp$. If $\varphi, \psi$ are modal mu-formulas then so are $\neg \varphi, \varphi \wedge \psi, \varphi \vee \psi, \nabla \varphi, \square \varphi$, and if $X \in \mathcal{V}$ and every free occurrence of $X$ in $\varphi$ is positive (in the scope of an even number of negations), then $\mu X \varphi$ and $\nu X \varphi$ are modal mu-formulas. We use the usual abbreviations $\rightarrow, \leftrightarrow$. An occurrence of $X$ in $\varphi$ is said to be bound if it is in the scope of a $\mu X$ or $\nu X$, and free, otherwise. For convenience, occurrences of propositional atoms will also be called 'free' occurrences. A sentence is a modal mu-formula with no free fixed point variables.

We write $\varphi\left(p_{1}, \ldots, p_{n}, X_{1}, \ldots, X_{m}\right)$ to indicate that the atoms and free variables in $\varphi$ are among $p_{1}, \ldots, p_{n}$ and $X_{1}, \ldots, X_{m}$, respectively. It will be implicit that $p_{1}, \ldots, p_{n}, X_{1}, \ldots, X_{m}$ are pairwise distinct. For modal mu-formulas $\varphi$ and $\psi$, and $\xi \in \mathcal{P} \cup \mathcal{V}, \varphi(\psi / \xi)$ denotes what we get by replacing all free occurrences of $\xi$ in $\varphi$ by $\psi$.

A frame is a pair $\mathcal{F}=(W, R)$, where $W$ is a non-empty set and $R \subseteq W \times W$. An assignment into $\mathcal{F}$ is a map $h: \mathcal{P} \cup \mathcal{V} \rightarrow \wp(W)$. For $\xi \in \mathcal{P} \cup \mathcal{V}$ and $U \subseteq W$, we write $h_{\xi}^{U}$ for the assignment that agrees with $h$ on all symbols other than $\xi$ and whose value on $\xi$ is $U$. We define $\llbracket \varphi \rrbracket_{h} \subseteq W$ by induction on $\varphi$; the frame $\mathcal{F}$ is implicit in the notation. For $\varphi \in \mathcal{P} \cup \mathcal{V}$ we put $\llbracket \varphi \rrbracket_{h}=h(\varphi)$. $\llbracket \top \rrbracket_{h}=W$, and $\llbracket \perp \rrbracket_{h}=\emptyset$. We put $\llbracket \neg \varphi \rrbracket_{h}=W \backslash \llbracket \varphi \rrbracket_{h}, \llbracket \varphi \wedge \psi \rrbracket_{h}=\llbracket \varphi \rrbracket_{h} \cap \llbracket \psi \rrbracket_{h}$, $\llbracket \varphi \vee \psi \rrbracket_{h}=\llbracket \varphi \rrbracket_{h} \cup \llbracket \psi \rrbracket_{h}, \llbracket \nabla \varphi \rrbracket_{h}=\left\{a \in W: \exists b\left(R(a, b) \wedge b \in \llbracket \varphi \rrbracket_{h}\right)\right\}$, and $\llbracket \square \varphi \rrbracket_{h}=\left\{a \in W: \forall b\left(R(a, b) \rightarrow b \in \llbracket \varphi \rrbracket_{h}\right)\right\}$. Finally, for a mu-formula $\varphi$ and $X \in \mathcal{V}$ with only positive free occurrences in $\varphi$, we note that the map $f: \wp(W) \rightarrow \wp(W)$ given by $f(U)=\llbracket \varphi \rrbracket_{h_{X}^{U}}$ is monotonic (this can be proved by induction on $\varphi$ ), and define

$$
\begin{aligned}
& \llbracket \mu X \varphi \rrbracket_{h}=\bigcap\left\{U \subseteq W: \llbracket \varphi \rrbracket_{h_{X}^{U}} \subseteq U\right\} \\
& \llbracket \nu X \varphi \rrbracket_{h}=\bigcup\left\{U \subseteq W: \llbracket \varphi \rrbracket_{h_{X}^{U}} \supseteq U\right\} .
\end{aligned}
$$

By the Knaster-Tarski theorem [24], these are (respectively) the least and greatest fixed points of $f$. As alternative notation, for a mu-formula $\varphi$ we write $(\mathcal{F}, h), a \models \varphi$ iff $a \in \llbracket \varphi \rrbracket_{h}$.

Let $\varphi$ be any modal mu-formula. It can be checked by induction that if $S \subseteq \mathcal{P} \cup \mathcal{V}$ and no $\xi \in S$ occurs free in $\varphi$, then $\llbracket \varphi \rrbracket_{g}=\llbracket \varphi \rrbracket_{h}$ for all assignments $g, h$ into the same frame that agree except perhaps on symbols in $S$. We say that $\varphi$ is positive (negative) if every atom and free fixed point variable in $\varphi$ occurs under an even (odd) number of negations. Suppose that $\pi$ is positive and $\gamma$ negative. It can be checked by induction that $\pi$ is monotonic and $\gamma$ antitonic: 
that is, if $h, h^{\prime}$ are assignments into the same frame and $h(\xi) \subseteq h^{\prime}(\xi)$ for all $\xi \in \mathcal{P} \cup \mathcal{V}$, then $\llbracket \pi \rrbracket_{h} \subseteq \llbracket \pi \rrbracket_{h^{\prime}}$ and $\llbracket \gamma \rrbracket_{h^{\prime}} \subseteq \llbracket \gamma \rrbracket_{h}$.

We say that $\varphi$ is valid in a frame $\mathcal{F}=(W, R)$ if $\llbracket \varphi \rrbracket_{h}=W$ for every assignment $h$ into $\mathcal{F}$, and valid if it is valid in every frame. We let ' $\equiv$ ' denote logical equivalence: $\varphi \equiv \psi$ iff $\varphi \leftrightarrow \psi$ is valid.

The dual operators to $\wedge, \vee, \square, \diamond, \mu, \nu$ are $\vee, \wedge, \diamond, \square, \nu, \mu$, respectively. As well as the usual $\neg(\varphi \wedge \psi) \equiv \neg \varphi \vee \neg \psi, \neg \square \varphi \equiv \diamond \neg \varphi$, etc, it can be checked that $\neg \mu X \varphi(X) \equiv \nu X \neg \varphi(\neg X / X)$ and $\neg \nu X \varphi(X) \equiv \mu X \neg \varphi(\neg X / X)$.

\subsection{First-order logic plus fixed points (FO+LFP)}

We will be very brief here, since first-order logic plus fixed point operators is a well known and well understood system. We broadly follow [11] and we refer the reader to this for much more information. We will use 'FO+LFP' to stand for first-order logic augmented by least and also greatest fixed point operators. We work in the signature with a binary relation symbol $R$ and unary relation symbols $P, X$ for each $p \in \mathcal{P}$ and $X \in \mathcal{V}$. The atomic formulas of FO+LFP are $x=y, R(x, y), \top, \perp, P(x)$, and $X(x)$, for any variables $x, y$, and $p \in \mathcal{P}, X \in \mathcal{V}$. If $\varphi, \psi$ are formulas then so are $\neg \varphi, \varphi \wedge \psi, \varphi \vee \psi, \forall x \varphi$, and $\exists x \varphi$. If $\varphi$ is a formula, $x$ a variable, and $S$ a unary relation symbol (arising either from $\mathcal{P}$ or $\mathcal{V}$ ) all of whose free occurrences in $\varphi$ are positive, then $[\operatorname{LFP}(S, x) \varphi]$ and $[\operatorname{GFP}(S, x) \varphi]$ are formulas with the same free first-order variables as $\varphi$, but in which $S$ is now bound. The semantics is as usual; in particular, if all free occurrences of $S$ in $\varphi\left(x, y_{1}, \ldots, y_{n}, S\right)$ are positive, then $M \models[\operatorname{LFP}(S, x) \varphi]\left(a, b_{1}, \ldots, b_{n}\right)$ iff $a$ is in the least fixed point of the (monotone) map $f: \wp M \rightarrow \wp M$ given by $f(U)=\left\{c \in M: M=\varphi\left(c, b_{1}, \ldots, b_{n}, U\right)\right\}$. Semantics of $[G F P(S, x) \varphi]$ are defined similarly, using greatest fixed points. Occasionally we will take fixed points of higher-arity relations.

We will also be taking 'simultaneous' fixed points. For more information, see $[11, \S 8.1-8.2]$. Let $\varphi_{i}\left(x_{i}, y_{1}, \ldots, y_{n}, S_{1}, \ldots, S_{m}\right)(1 \leq i \leq m)$ be FO+LFPformulas positive in $S_{1}, \ldots, S_{m}$. Fix a structure $M$ and $b_{1}, \ldots, b_{n} \in M$. For each $1 \leq i \leq m$ define $F^{i}\left(S_{1}, \ldots, S_{m}\right)=\left\{c \in M: M=\varphi_{i}\left(c, b_{1}, \ldots, b_{n}, S_{1}, \ldots, S_{m}\right)\right\}$, for $S_{1}, \ldots, S_{m} \subseteq M$. Let $\left(T_{1}, \ldots, T_{m}\right)$ be the simultaneous least fixed point of the sequence $\left(F^{1}, \ldots, F^{m}\right)$ of maps. Very slightly varying [11]'s notation, we then write

$$
M \models\left[S-L F P\left(i, x_{1}, S_{1}, \ldots, x_{m}, S_{m}\right) \varphi_{1}, \ldots, \varphi_{m}\right]\left(a, b_{1}, \ldots, b_{n}\right)
$$

if $a \in T_{i}$. This is expressible in standard FO+LFP.

As in the mu-calculus, $\equiv$ will denote the relation of logical equivalence. Any formula positive in $P$ is monotonic in $P$ as well.

\subsection{Standard translations}

For a first-order variable $x$, every modal mu-formula $\varphi\left(p_{1}, \ldots, p_{n}, X_{1}, \ldots, X_{m}\right)$ has a standard translation $S T_{x}(\varphi)$ : a formula $\varphi^{\prime}\left(x, P_{1}, \ldots, P_{n}, X_{1}, \ldots, X_{m}\right)$ of $\mathrm{FO}+\mathrm{LFP}$ defined as follows: 
1. $S T_{x}(p)=P(x), S T_{x}(X)=X(x), S T_{x}(\top)=\top$, and $S T_{x}(\perp)=\perp$,

2. $S T_{x}(\neg \varphi)=\neg S T_{x} \varphi, S T_{x}(\varphi \wedge \psi)=S T_{x}(\varphi) \wedge S T_{x}(\psi)$, and $S T_{x}(\varphi \vee \psi)=$ $S T_{x}(\varphi) \vee S T_{x}(\psi)$,

3. $S T_{x}(\nabla \varphi)=\exists y\left(R(x, y) \wedge S T_{y}(\varphi)\right)$, for some variable $y \neq x$,

4. $S T_{x}(\square \varphi)=\forall y\left(R(x, y) \rightarrow S T_{y}(\varphi)\right)$, for some variable $y \neq x$,

5. $S T_{x}(\mu X \varphi)=\left[\operatorname{LFP}(X, x) S T_{x} \varphi\right]$,

6. $S T_{x}(\nu X \varphi)=\left[G F P(X, x) S T_{x} \varphi\right]$.

For any frame $\mathcal{F}=(W, R)$, any assignment $h$ into $\mathcal{F}$, any $a \in W$, and any modal mu-formula $\varphi\left(p_{1}, \ldots, p_{n}, X_{1}, \ldots, X_{m}\right)$ with $S T_{x} \varphi=\varphi^{\prime}\left(x, P_{1}, \ldots, P_{n}, X_{1}, \ldots\right.$, $\left.X_{m}\right)$, we have $(\mathcal{F}, h), a \models \varphi$ iff $\mathcal{F} \models \varphi^{\prime}\left(a, h\left(p_{1}\right), \ldots, h\left(p_{n}\right), h\left(X_{1}\right), \ldots, h\left(X_{m}\right)\right)$. Note that if $\varphi$ is positive in $p_{i}$ then $S T_{x}(\varphi)$ is positive in $P_{i}$.

\section{Sahlqvist's theorem and the mu-calculus}

Here we will describe the existing work that led us to the position recorded in this paper.

\subsection{Classical Sahlqvist correspondence}

Sahlqvist formulas originated in [22]. In spite of (or perhaps because of) their importance in modal logic today, there seems to be no universally agreed modern definition of them. We will adopt the following simple definition.

DEFINITION 3.1 [Sahlqvist formula]

1. Any positive formula is a Sahlqvist formula.

2. Any formula of the form $\neg \square^{n} s$ (a negated 'boxed atom') is a Sahlqvist formula, where $n \geq 0, \square^{0} \varphi=\varphi, \square^{n+1} \varphi=\square\left(\square^{n} \varphi\right)$, and $s$ is a propositional atom.

3. If $\varphi, \psi$ are Sahlqvist formulas then so are $\varphi \vee \psi$ and $\square \varphi$.

Many commonly arising modal axioms are equivalent to Sahlqvist formulas. To illustrate, the formula $\square p \rightarrow p$ is equivalent to $\neg \square p \vee p$, which is constructed from the negated boxed atom $\neg \square p$ (clause 2) and the positive formula $p$ (clause 1 ) using $\vee$ (clause 3 ). It is common to include $\varphi \wedge \psi$ in clause 3 above - for example, the definition of Sahlqvist formulas in [7, definition 3.51] boils down to this. We do not allow $\wedge$ in clause 3 for two reasons. First, any formula obtained by adding $\wedge$ to clause 3 is in any case equivalent to a conjunction of Sahlqvist formulas as defined above, because any occurrence of $\wedge$ can be moved up through the $\vee_{s}$ and $\square$ s using distributivity. Second, the argument coming up in a moment is simpler without $\wedge$ in clause 3 . But when we come to Sahlqvist mu-formulas, we will want to include $\wedge$.

Sahlqvist formulas have two key properties: 
Correspondence. For any Sahlqvist formula $\varphi$, there is a first-order sentence $\chi_{\varphi}$, called the frame correspondent of $\varphi$, that is true in an arbitrary Kripke frame iff $\varphi$ is valid in that frame. Moreover, $\chi_{\varphi}$ can be computed from $\varphi$ by a simple algorithm. A stronger 'local correspondent' expressing validity of $\varphi$ at a given world is also obtained. This can also be done in our work, but we leave it to the reader.

Completeness. For any Sahlqvist formula $\varphi$, the basic modal logic $K$ augmented with $\varphi$ as an extra axiom is sound and complete for the class of frames defined by $\chi_{\varphi}$.

These properties are of course related, and further algebraic properties of Sahlqvist formulas have been established (e.g., [15]). The celebrated 'Esakia lemma' [12] is used in a key step in the proof of completeness (e.g., [23]). In this paper we are concerned only with correspondence, and we confine our discussion to that topic. There are several proofs of Sahlqvist correspondence in the literature: e.g., $[22,2,23,7]$. But the idea can be simply explained, as follows. It will be familiar to many readers, but we (briefly) go through the steps because we intend to generalise them later.

Let $\varphi$ be a Sahlqvist formula and $\mathcal{F}=(W, R)$ a Kripke frame.

Step 1. Assume that $\varphi$ is not valid in $\mathcal{F}$. This says that there is a model $\mathcal{M}=(\mathcal{F}, h)$, for some assignment $h$ of atoms into $\mathcal{F}$, and some world $a \in W$, such that $\mathcal{M}, a \models \neg \varphi$. Now $\neg \varphi$ is plainly equivalent to a formula of the form

$$
\sigma\left(\gamma_{1}, \ldots, \gamma_{m}, \beta_{1}, \ldots, \beta_{n}\right)
$$

where $\sigma\left(p_{1}, \ldots, p_{m}, q_{1}, \ldots, q_{n}\right)$ is a formula made from distinct atoms $p_{1}, \ldots$, $p_{m}, q_{1}, \ldots, q_{n}$ using only $\wedge$ and $\diamond$ (the duals of the operations in clause 3 of definition 3.1); each of $q_{1}, \ldots, q_{n}$ occurs exactly once in $\sigma ; \gamma_{1}, \ldots, \gamma_{m}$ are negative formulas; $\beta_{1}, \ldots, \beta_{n}$ are boxed atoms; and (1) is shorthand for the result

$$
\sigma\left(\gamma_{1} / p_{1}, \ldots, \gamma_{m} / p_{m}, \beta_{1} / q_{1}, \ldots, \beta_{n} / q_{n}\right)
$$

of simultaneously replacing each atom $p_{i}$ in $\sigma$ by $\gamma_{i}$ and each $q_{j}$ by $\beta_{j}$. So $\varphi$ is not valid in $\mathcal{F}$ iff there are $a, h$ with

$$
(\mathcal{F}, h), a \models \sigma\left(\gamma_{1}, \ldots, \gamma_{m}, \beta_{1}, \ldots, \beta_{n}\right) .
$$

Step 2. Now we observe the following critical fact. Let $x$ be any first-order variable.

LEMMA 3.2 The standard translation $S T_{x}\left(\sigma\left(p_{1}, \ldots, p_{m}, q_{1}, \ldots, q_{n}\right)\right)$ of $\sigma$ is equivalent to a formula $\sigma^{\prime}\left(x, P_{1}, \ldots, P_{m}, Q_{1}, \ldots, Q_{n}\right)$ of the form

$$
\exists y_{1} \ldots y_{n}\left(\psi\left(x, P_{1}, \ldots, P_{m}, \bar{y}\right) \wedge \bigwedge_{1 \leq j \leq n} Q_{j}\left(y_{j}\right)\right),
$$

for some first-order formula $\psi\left(x, P_{1}, \ldots, P_{m}, \bar{y}\right)$ positive in each of $P_{1}, \ldots, P_{m}$, where $\bar{y}=\left(y_{1}, \ldots, y_{n}\right)$ is a tuple of distinct variables different from $x$. 
The proof is a simple induction on the structure of $\sigma$, and it can be done precisely because (as a result of clause 3 of definition 3.1) $\sigma$ only involves $\wedge$ and $\diamond$, and each $q_{j}$ occurs exactly once in $\sigma$. If we allowed $\wedge$ in clause $3, \sigma^{\prime}$ would be more complicated: a disjunction of formulas of the form (3).

With (3) at hand, we see that (2) literally says that for some $a, h$,

(*) there are $b_{1}, \ldots, b_{n} \in W$, standing in a certain relation to $a$ and to each other specified by $\psi$ (formally, by $\mathcal{F} \models \psi\left(a, \llbracket \gamma_{1} \rrbracket_{h}, \ldots, \llbracket \gamma_{m} \rrbracket_{h}, b_{1}, \ldots, b_{n}\right)$ ), and such that $(\mathcal{F}, h), b_{j} \models \beta_{j}$ for each $1 \leq j \leq n$.

Step 3. The next critical step is to observe that without loss of generality we can replace $h$ by a 'minimal assignment' $h^{\circ}$, satisfying $h^{\circ}(s) \subseteq h(s)$ for every atom $s$ occurring in $\varphi$. In fact, $h^{\circ}$ is the assignment where each $h^{\circ}(s)$ is as small as possible subject to the condition that $\left(\mathcal{F}, h^{\circ}\right), b_{j}=\beta_{j}$ for $1 \leq j \leq n$. The definition of $h^{\circ}$ is uniform in $b_{1}, \ldots, b_{n}$.

To find $h^{\circ}$, for each atom $s$ we collect up all the boxed atoms $\beta_{j}$ involving $s$. To illustrate, suppose that there are just two of them: $\beta_{3}=\square^{2} s$, and $\beta_{7}=$ $\square^{0} s=s$. (So $\beta_{1}, \beta_{5}$, etc., are boxed atoms involving other atoms than $s$.) Then (*) states that $(\mathcal{F}, h), b_{3} \models \square^{2} s$ and $(\mathcal{F}, h), b_{7} \models s$. This will be preserved if we replace $h$ by an assignment $g$ with $g(s)=\left\{w \in W: \mathcal{F} \models \exists z\left(R\left(b_{3}, z\right) \wedge\right.\right.$ $R(z, w))\} \cup\left\{b_{7}\right\}$. This is the 'minimal' assignment satisfying $(\mathcal{F}, g), b_{3} \models \square^{2} s$ and $(\mathcal{F}, g), b_{7} \models s$. Any assignment $g^{\prime}$ making $\beta_{3}$ true at $b_{3}$ and $\beta_{7}$ at $b_{7}$ must plainly satisfy $g(s) \subseteq g^{\prime}(s)$, and in particular, we have $g(s) \subseteq h(s)$. Let $h^{\circ}$ be the 'minimal assignment' that assigns the minimal value $g(s)$ to each atom $s$ as just explained. If $s$ does not occur in any $\beta_{j}$ then $h^{\circ}(s)=\emptyset$.

Now $h^{\circ}(s) \subseteq h(s)$ for all atoms $s$. Consequently, by antitonicity of negative formulas, $\llbracket \gamma_{i} \rrbracket_{h} \subseteq \llbracket \gamma_{i} \rrbracket_{h}$ 。 for $i=1, \ldots, m$. Since $P_{1}, \ldots, P_{m}$ occur only positively in $\psi$, the truth of $\psi\left(a, \llbracket \gamma_{1} \rrbracket_{h}, \ldots, \llbracket \gamma_{m} \rrbracket_{h}, b_{1}, \ldots, b_{n}\right)$ in $(*)$ is unaffected by our replacing $h$ by $h^{\circ}$.

So if $(*)$ holds for some assignment $h$, then it holds for $h^{\circ}$. Since if $(*)$ holds for $h^{\circ}$ then it certainly holds for some $h$, we conclude that $\varphi$ is not valid in $\mathcal{F}$ iff $(*)$ holds for some $a$ and for $h^{\circ}$.

We now make one final observation: it is automatic that $\left(\mathcal{F}, h^{\circ}\right), b_{j}=\beta_{j}$ for each $1 \leq j \leq n$, since $h^{\circ}$ is defined precisely to achieve this. We conclude that $\varphi$ is not valid in $\mathcal{F}$ iff:

$(* *)$ there are $a, b_{1}, \ldots, b_{n} \in W$ with $\mathcal{F} \models \psi\left(a, \llbracket \gamma_{1} \rrbracket_{h^{\circ}}, \ldots, \llbracket \gamma_{m} \rrbracket_{h^{\circ}}, b_{1}, \ldots, b_{n}\right)$, where $h^{\circ}$ is defined as above.

Step 4. The final critical step is to notice that for each atom $s$, the value $h^{\circ}(s)$ is first-order definable with the parameters $b_{1}, \ldots, b_{n}$. We have

$$
h^{\circ}(s)=\left\{c \in W: \mathcal{F} \models \delta_{s}\left(c, b_{1}, \ldots, b_{n}\right)\right\},
$$

where $\delta_{s}\left(x, y_{1}, \ldots, y_{n}\right)$ is a certain first-order formula in the frame language, and one that we can explicitly construct. In the example above, we had $h^{\circ}(s)=$ 
$\left\{c \in W: \mathcal{F} \models \exists z\left(R\left(b_{3}, z\right) \wedge R(z, c)\right)\right\} \cup\left\{b_{7}\right\}$ - this is definable as $\{c \in W:$ $\left.\mathcal{F} \models \delta_{s}\left(c, b_{1}, \ldots, b_{n}\right)\right\}$, where

$$
\delta_{s}\left(x, y_{1}, \ldots, y_{n}\right)=\exists z\left(R\left(y_{3}, z\right) \wedge R(z, x)\right) \vee x=y_{7} .
$$

Summing up. In the light of $(* *)$ and step 4 , we see that $\varphi$ is not valid in $\mathcal{F}$ iff

$$
\mathcal{F} \models \exists x \bar{y} \theta(x, \bar{y}),
$$

where $\theta$ denotes the result of replacing each subformula of $\psi\left(x, P_{1}, \ldots, P_{m}, \bar{y}\right)$ of the form $P_{i}(t)$ (for some $1 \leq i \leq m$ and some variable $t$ ) by: the formula obtained from $S T_{t}\left(\gamma_{i}\right)$ by replacing each subformula $S(v)$ (for an atom $s$ and a variable $v$ ) by $\delta_{s}\left(v / x, y_{1}, \ldots, y_{n}\right)$ (which is the definition of $h^{\circ}(s)$ ). By construction, (4) means exactly the same as $(* *)$ and is equivalent to $\varphi$ 's failing to be valid in $\mathcal{F}$. Consequently, the negation $\forall x \bar{y} \neg \theta(x, \bar{y})$ of the first-order sentence in (4) is our desired frame correspondent for $\varphi$.

We would like to generalise this argument, eventually to the mu-calculus.

\subsection{PIA formulas}

In [4], van Benthem showed how to generalise steps 3 and 4 to a wider class of modal formulas than boxed atoms, at the cost of ending up with a frame correspondent not in first-order logic but in FO+LFP: first-order logic plus the least and greatest fixed point operators.

What step 3 needs is the existence of a minimal assignment that makes a formula $\beta$ true at a given world $y$ of a Kripke frame, given that there exists at least one assignment making $\beta$ true at $y$. As we saw, if $\beta$ is a boxed atom $\square^{d} s$ then there is indeed a minimal assignment to $s$, namely, $\{w \in W: \mathcal{F} \models$ $\left.R^{d}(y, w)\right\}$, where $R^{0}(y, w)$ is $y=w$ and $R^{d+1}(y, w)$ is $\exists z\left(R(y, z) \wedge R^{d}(z, w)\right)$.

[4] studied first-order sentences $\varphi(S)$ (for a unary relation symbol $S$ corresponding to the atom $s$ ) that admit such a minimal assignment, in the sense that in any first-order structure $M$ there is a minimal $S \subseteq M$ with $M \models \varphi(S)$. It was shown that a sufficient condition for $\varphi(S)$ to admit a minimal assignment is that it has the intersection property $(I P)$ : namely, that for any $M$, index set $I$, and subsets $S_{i} \subseteq M(i \in I)$, if $M \mid=\varphi\left(S_{i}\right)$ for each $i \in I$ then $M \models \varphi\left(\bigcap_{i \in I} S_{i}\right)$. The minimal assignment to $S$ that makes $\varphi(S)$ true is then simply $\bigcap\{S \subseteq M: M \models \varphi(S)\}$. It was also proved that $\varphi(S)$ has IP iff it is equivalent to a sentence of the form

$$
\forall y(\psi(S, y) \rightarrow S(y)),
$$

where $\psi(S, y)$ is positive in $S$. Such sentences have the form 'positive implies atomic', or for short, 'PIA'.

This is for first-order logic, and no similar characterisation of the modal version of IP was given. Nonetheless, [4] did exhibit a modal analogue of 'PIA implies IP implies minimal assignment exists'. This arises by considering modal 
formulas $\varphi(s)$ that we will call semantically PIA formulas, whose standard translations $S T_{x}(\varphi(s))$ are equivalent to PIA formulas of the form

$$
\forall y(\psi(S, x, y) \rightarrow S(y)),
$$

for $\psi$ positive in $S$. Boxed atoms are examples: $S T_{x}\left(\square^{d} s\right) \equiv \forall y\left(R^{d}(x, y) \rightarrow\right.$ $S(y)$ ), which is of the required form (5). But there are many more. First, any atom $s$ is a semantically PIA formula, since its standard translation $S T_{x}(\varphi)$ is $S(x)$ - this is equivalent to $\forall y(y=x \rightarrow S(y))$, which is of the form (5). Second, it can be verified that the semantically PIA formulas $\varphi(s)$, for a fixed atom $s$, are closed under $\wedge$ and $\square$ (though not under $\vee$ ). Third, if $\varphi(s)$ is semantically PIA and $\pi(s)$ is positive in $s$ then $\pi(s) \rightarrow \varphi(s)$ is also semantically PIA. Since for Sahlqvist purposes we would like a syntactically defined class of semantically PIA formulas, we say that a modal formula $\varphi(s)$ is syntactically PIA if it is obtained from $s$ by applying $\wedge, \square$, and $\pi(s) \rightarrow \cdot$, where $\pi(s)$ is positive in $s$. Boxed atoms are plainly (very) special cases of syntactically PIA formulas. By the above, every syntactically PIA formula is semantically PIA. Any syntactically PIA formula, and indeed any semantically PIA formula, admits a minimal assignment to $s$ as required by step 3 of the correspondence proof in section 3.1.

For step 4, we also need that the minimal assignment is definable in firstorder logic. The minimal $S$ satisfying (5) need not be first-order definable. However, it is definable in FO+LFP. This is because the minimal $S$ satisfying (5) (in a frame $\mathcal{F}=(W, R)$, for a given $x \in W$ ) is the intersection of all $S$ satisfying (5). By the Knaster-Tarski theorem, this intersection is the least fixed point of the monotone map $f_{\psi, x}: \wp(W) \rightarrow \wp(W)$ given by $f_{\psi, x}(S)=\{a \in$ $W: \mathcal{F}=\psi(S, x, a)\}$, for $S \subseteq W$. It is therefore defined by the FO+LFP-formula $[\operatorname{LFP}(S, y) \psi](x, y)$.

The astute reader will have noticed that step 3 also required that we can take the union of the minimal assignments to a given atom $s$ from all of the boxed atoms $\beta_{j}$ involving $s$, obtaining a single (definable) minimal assignment that still satisfies all these $\beta_{j}$. This is true for syntactically PIA formulas, for much the same reason that they are closed under $\wedge$, but properly it is a consideration for the 'clause 3' structure of the Sahlqvist formula.

We conclude that we can allow negated syntactically PIA formulas in clause 2 of definition 3.1, if we do not mind the frame correspondent being in FO+LFP instead of first-order logic.

\subsection{PIA mu-calculus formulas}

The main contribution of the current paper now begins. As suggested in [4], if we are willing to admit frame correspondents in $\mathrm{FO}+\mathrm{LFP}$, why not go further and consider formulas of the modal mu-calculus, whose standard translations automatically lie in this language? Let us say that a modal mu-calculus formula $\beta(s)$ is semantically PIA if its standard translation $S T_{x}(\beta)$ is equivalent to a 
FO+LFP-formula of the form

$$
\forall y(\psi(S, x, y) \rightarrow S(y)),
$$

where $\psi$ is positive in $S$. There will always be a FO+LFP-definable minimal assignment to $s$ making $\beta$ true at a world $a$ in a frame $\mathcal{F}$, namely, $\{c: \mathcal{F} \models$ $\left.\psi^{\prime}(a, c)\right\}$, where $\psi^{\prime}(x, y)=[L F P(S, y) \psi](x, y)$.

This definition of PIA formula is semantic. As before, we now have the task of defining a wide syntactic class of semantically PIA mu-formulas. Starting from an atom $s$ and fixed-point variables, we can close under $\wedge, \square$, and $\pi(s) \rightarrow \cdot$ as before, where $\pi(s)$ is now a modal mu-sentence positive in $s$. As we will see below (section 4 ), we can also close under the greatest fixed point operator $\nu$. Any sentence $\varphi(s)$ obtained using these four operations admits a minimal assignment to $s$ that makes $\varphi$ true at a world $x$ of a frame; the minimal assignment is definable in FO+LFP. So we could allow the negations of such formulas in clause 2 of definition 3.1.

We can even go further and handle several atoms at once (cf. $[4, \S 4.4])$. It will be shown that if $\beta\left(s_{1}, \ldots, s_{t}\right)$ is any sentence obtained from atoms and fixed point variables using $\wedge, \square, \nu$, and $\pi \rightarrow \cdot$ for a positive modal mu-sentence $\pi$, then $S T_{x}(\beta)$ is equivalent to $\bigwedge_{k=1}^{t} \forall y_{k}\left(\psi_{k}\left(S_{1}, \ldots, S_{t}, x, y_{k}\right) \rightarrow S_{k}\left(y_{k}\right)\right)$ for some FO+LFP-formulas $\psi_{1}, \ldots, \psi_{t}$ positive in $S_{1}, \ldots, S_{t}$. We can then extract a minimal assignment to $S_{1}, \ldots, S_{t}$ using simultaneous fixed points, which are well known to be expressible in FO+LFP: see $\S 2.2$.

\subsection{Clause 1}

In step 3 of the correspondence proof, we noted that the negative formulas kept their truth values when we replaced the original assignment $h$ by the minimal one, $h^{\circ}$. All that was needed for this was antitonicity, which still holds if we allow positive mu-calculus formulas in clause 1 of definition 3.1.

\subsection{Clause 3}

Sahlqvist formulas were defined as the closure of positive formulas and negated boxed atoms under $\vee, \square$. We have seen how we can generalise boxed atoms (to PIA mu-formulas) and positive formulas (to positive mu-calculus formulas). Now we would like to generalise the 'clause 3' structure: the closure operations $\vee, \square$.

All we required of these operations was that, when dualised to $\wedge, \diamond$, they allow lemma 3.2 to be proved. If we include $\vee$ here as well, a form of the lemma involving a disjunction of formulas of the form (3) can be proved. We would like to add $\mu$, and to leverage this powerful operator we would like to have both $\wedge$ and $\vee$ available. (For example, we can already express $\diamond(p \wedge q)$ using $\wedge$ and $\diamond$, so we would like to express its 'reflexive transitive closure' version $\diamond^{*}(p \wedge q)$, by $\sigma_{1}(p, q)=\mu X((p \wedge q) \vee \diamond X)$. This requires $\wedge$ and $\vee$. $)$ 
It turns out that a disjunctive form of lemma 3.2 can be proved for any formula $\sigma\left(p_{1}, \ldots, p_{m}, q_{1}, \ldots, q_{n}\right)$ built using only $\vee, \diamond, \mu$, where the formula $\psi$ in (3) is now in FO+LFP of course.

To allow $\wedge$ as well, we have to make restrictions. For example, the standard translation $S T_{x}\left(\sigma_{2}\right)$ of the formula $\sigma_{2}\left(q_{1}, q_{2}\right)=\mu X\left(q_{1} \vee\left(q_{2} \wedge \diamond X\right)\right)$, expressing ' $q_{2}$ until $q_{1}$ ', is not equivalent to a disjunction of formulas of the form $\exists y_{1} y_{2}\left(\psi(x, y, z) \wedge Q_{1}\left(y_{1}\right) \wedge Q_{2}\left(y_{2}\right)\right)$ given in (3). A sufficient restriction is to allow $\sigma \wedge \tau$ only if (i) $\sigma$ and $\tau$ have no atoms from $q_{1}, \ldots, q_{n}$ (corresponding to the boxed atoms) in common, and (ii) if either has a free fixed point variable then the other is a sentence not involving $q_{1}, \ldots, q_{n}$. This restriction allows $\sigma_{1}$ but not $\sigma_{2}$.

Now lemma 3.2 was only a tool for the correspondence proof. What is the effect of the restrictions on $\wedge$ in $\sigma$ on actual Sahlqvist formulas? The effect of (i) is nil, since we can meet it by simply using fresh atoms in $\tau$ - this doesn't matter since in (1) we substitute formulas for the atoms of $\sigma$ anyway. The effect of (ii) is that for $\varphi \vee \psi$ to be a Sahlqvist formula, if one of $\varphi, \psi$ is not a sentence then the other must be a sentence not involving any negated boxed atoms/PIA formulas - i.e., a positive sentence.

The 'reason' why lemma 3.2 can be proved for such formulas $\sigma$ is that they are completely additive in each $q_{k}$. Formally, if $\mathcal{F}$ is a frame, $h_{i}(i \in I \neq \emptyset)$ are assignments into $\mathcal{F}$ that agree on all atoms other than $q_{k}$, and $h$ is the assignment given by $h(p)=\bigcup_{i \in I} h_{i}(p)$ for each atom $p$, then for any world $a$ of $\mathcal{F}$ we have $(\mathcal{F}, h), a \models \sigma$ iff $\left(\mathcal{F}, h_{i}\right), a \models \sigma$ for some $i \in I$. The restrictions on $\wedge$ are to ensure that this holds.

Suppose for example that $\sigma$ only involves the atom $q$, and $S T_{x}(\sigma)=\psi(x, Q)$, say. Let $\psi_{0}(x), \psi_{1}(x, y)$ denote the result of replacing each subformula $Q(v)$ of $\psi$ by $\perp$ and $v=y$, respectively. Then by complete additivity,

$$
S T_{x}(\sigma) \equiv \psi_{0}(x) \vee \exists y\left(\psi_{1}(x, y) \wedge Q(y)\right) .
$$

This form is close enough to (3) for the correspondence proof to work. If $\sigma$ involves multiple atoms, the argument can be iterated. So we can replace clause 3 of definition 3.1 by a construction allowing (the duals of) $\vee, \diamond, \mu$, and the restricted $\wedge$ as just explained.

The trouble-maker is clearly $\wedge$. If whenever $\wedge$ is used in $\sigma\left(p_{1}, \ldots, p_{m}, q_{1}, \ldots\right.$, $q_{n}$ ), one of the conjuncts is a sentence not involving $q_{1}, \ldots, q_{n}$, then we can prove a stronger form of complete additivity. Passing to the dual operations $\wedge, \square, \nu$, this becomes a strong form of 'complete multiplicativity' analogous to the intersection property (IP), which we will use to show that $\wedge, \square, \nu$ and $P O S \rightarrow \cdot$ can be applied to PIA formulas with multiple atoms while preserving the existence of a definable minimal assignment.

\subsection{Sahlqvist formulas in the mu-calculus}

Let us formalise the position we have arrived at. All formulas below are of the modal mu-calculus. 
DEFINITION 3.3 [PIA formulas] We define the PIA formulas as follows.

1. Any atom is a PIA formula.

2. Any fixed point variable is a PIA formula.

3. If $\beta, \gamma$ are PIA formulas then so are $\beta \wedge \gamma, \square \beta$, and $\nu X \beta$ (for any fixed point variable $X$ ).

4. If $\beta$ is a PIA formula and $\pi$ is a positive modal mu-sentence, then $\pi \rightarrow \beta$ is a PIA formula.

In the end we are only interested in PIA sentences. These may not look of the form 'positive implies atomic', but we will see that their standard translations are equivalent to conjunctions of formulas of this form, so we feel the term 'PIA' is justified.

DEFINITION 3.4 [Sahlqvist mu-formula]

1. Any positive sentence is a Sahlqvist mu-formula.

2. Any negated PIA sentence is a Sahlqvist mu-formula.

3. Any fixed point variable is a Sahlqvist mu-formula.

4. If $\varphi, \psi$ are Sahlqvist mu-formulas then so are $\varphi \wedge \psi, \square \varphi$, and $\nu X \varphi$ (for any fixed point variable $X$ ).

5. If $\varphi, \psi$ are Sahlqvist mu-formulas, and if one of them is not a sentence then the other is a positive sentence, then $\varphi \vee \psi$ is a Sahlqvist mu-formula.

A Sahlqvist mu-sentence is a Sahlqvist mu-formula that is a sentence.

In summary, a Sahlqvist mu-sentence is any sentence obtained by applying $\wedge, \square$, and $\nu$ to fixed point variables, positive sentences, and negated PIA sentences; $\checkmark$ can also be applied so long as if one of the disjuncts is not a sentence then the other is a positive sentence. See section 5 for examples. In the next section we will prove a correspondence theorem for Sahlqvist mu-sentences.

\section{Correspondence theorem}

This section contains the formal proofs of the paper. We will prove a correspondence theorem for Sahlqvist mu-sentences (theorem 4.13 below). The initial sections contain preliminaries. 


\subsection{Skeletons}

Our main technical tool will be formulas that we call skeletons, because they will support the negative formulas and PIA formulas (generalising the boxed atoms) in Sahlqvist formulas, as in (1). (In this role, they are analogous to the universal prefix that is extracted in the 'Sahlqvist-van Benthem algorithm' in [7]. Skeletons allow a richer Sahlqvist syntax, including, for example, negative formulas in antecedents $-\diamond(\neg p \wedge \square p) \rightarrow \cdots$ is fine.) We will also use them to show that our PIA formulas really are semantically PIA.

Recall that $\mathcal{P}$ is our fixed set of atoms, and $\mathcal{V}$ the set of fixed point variables.

DEFINITION 4.1 [Q-skeleton] Let $\mathcal{Q} \subseteq \mathcal{P}$ be arbitrary.

1. Any atomic mu-formula (i.e., an atom, a fixed point variable, $\top$, or $\perp$ ) is a $\mathcal{Q}$-skeleton.

2. If $\sigma, \tau$ are $\mathcal{Q}$-skeletons then so are $\sigma \vee \tau, \diamond \sigma$, and $\mu X \sigma$ (for any fixed point variable $X$ ).

3. If $\sigma$ is a $\mathcal{Q}$-skeleton and $\tau$ is a positive sentence involving no atoms from $\mathcal{Q}$, then $\sigma \wedge \tau$ and $\tau \wedge \sigma$ are $\mathcal{Q}$-skeletons.

REMARK 4.2 Any $\mathcal{Q}$-skeleton is a $\mathcal{Q}^{\prime}$-skeleton for every $\mathcal{Q}^{\prime} \subseteq \mathcal{Q}$ : increasing $\mathcal{Q}$ strengthens the restrictions on $\mathcal{Q}$-skeletons. However, if $\sigma$ is a $\mathcal{Q}$-skeleton and $\mathcal{Q}^{\prime}$ is a set of atoms not occurring in $\sigma$, a simple induction shows that $\sigma$ is a $\mathcal{Q} \cup \mathcal{Q}^{\prime}$-skeleton.

The main semantic property of skeletons is a form of complete additivity, as we will see in proposition 4.4. Fix a frame $\mathcal{F}=(W, R)$.

DEFINITION 4.3 Let $\mathcal{H}$ be a set of assignments into $\mathcal{F}$.

1. Write $\bigcup \mathcal{H}$ for the assignment $g$ given by $g(\xi)=\bigcup\{h(\xi): h \in \mathcal{H}\}$ for each atom or fixed point variable $\xi$.

2. Let $\mathcal{Q} \subseteq \mathcal{P}$ be a set of atoms. We say that $\mathcal{H}$ is $\mathcal{Q}$-variant if $h(p)=h^{\prime}(p)$ for all atoms $p \in \mathcal{P} \backslash Q$ and all $h, h^{\prime} \in \mathcal{H}$. (Important: there are no restrictions on the values of $h \in \mathcal{H}$ on fixed point variables.)

PROPOSITION 4.4 Fix $\mathcal{Q} \subseteq \mathcal{P}$. Let $\sigma$ be a $\mathcal{Q}$-skeleton and $\mathcal{H}$ a non-empty $\mathcal{Q}$-variant set of assignments into $\mathcal{F}$. Then $\llbracket \sigma \cup_{\mathcal{H}}=\bigcup\left\{\llbracket \sigma \rrbracket_{h}: h \in \mathcal{H}\right\}$.

Proof. We prove the proposition by induction on $\sigma$. We write $g=\bigcup \mathcal{H}$. If $\sigma \in \mathcal{P} \cup \mathcal{V}$ then $\llbracket \sigma \rrbracket_{g}=g(\sigma)=\bigcup\{h(\sigma): h \in \mathcal{H}\}=\bigcup\left\{\llbracket \sigma \rrbracket_{h}: h \in \mathcal{H}\right\}$. If $\sigma=\perp$, the result is trivial. If $\sigma=\top$, then because $\mathcal{H} \neq \emptyset$ we have $\llbracket \top \rrbracket_{g}=\bigcup\left\{\llbracket \top \rrbracket_{h}\right.$ : $h \in \mathcal{H}\}$.

We pass to the inductive steps. First suppose that $\sigma=\tau \vee \xi$, where $\tau, \xi$ are $\mathcal{Q}$-skeletons. Then $\llbracket \sigma \rrbracket_{g}=\llbracket \tau \rrbracket_{g} \cup \llbracket \xi \rrbracket_{g}$. By the induction hypothesis, $\llbracket \tau \rrbracket_{g} \cup \llbracket \xi \rrbracket_{g}=$ $\bigcup\left\{\llbracket \tau \rrbracket_{h}: h \in \mathcal{H}\right\} \cup \bigcup\left\{\llbracket \xi \rrbracket_{h}: h \in \mathcal{H}\right\}=\bigcup\left\{\llbracket \tau \rrbracket_{h} \cup \llbracket \xi \rrbracket_{h}: h \in \mathcal{H}\right\}=\bigcup\left\{\llbracket \sigma \rrbracket_{h}: h \in\right.$ $\mathcal{H}\}$. 
Next let $\sigma=\diamond \tau$ for some $\mathcal{Q}$-skeleton $\tau$. Let $w \in W$. Then $w \in \llbracket \sigma \rrbracket_{g}=\llbracket \triangleright \tau \rrbracket_{g}$ iff there is $v \in \llbracket \tau \rrbracket_{g}$ with $R(w, v)$. Inductively, $\llbracket \tau \rrbracket_{g}=\bigcup\left\{\llbracket \tau \rrbracket_{h}: h \in \mathcal{H}\right\}$. So the above holds iff there are $h \in \mathcal{H}$ and $v \in \llbracket \tau \rrbracket_{h}$ with $R(w, v)$. This is iff there is $h \in \mathcal{H}$ with $w \in \llbracket \triangleright \tau \rrbracket_{h}=\llbracket \sigma \rrbracket_{h}$ : i.e., iff $w \in \bigcup\left\{\llbracket \sigma \rrbracket_{h}: h \in \mathcal{H}\right\}$, as required.

Next suppose that $\sigma=\tau \wedge \xi$ for some $\mathcal{Q}$-skeleton $\tau$ and positive ${ }^{1}$ sentence $\xi$ involving no atom in $\mathcal{Q}$ (the case $\xi \wedge \tau$ is handled similarly). As $\mathcal{H}$ is $\mathcal{Q}$-variant, for each $h \in \mathcal{H}, g, h$ agree on all free symbols in $\xi$, and so $\llbracket \xi \rrbracket_{g}=\llbracket \xi \rrbracket_{h}$ for each $h \in \mathcal{H}$. Now $\llbracket \sigma \rrbracket_{g}=\llbracket \tau \rrbracket_{g} \cap \llbracket \xi \rrbracket_{g}$. By the induction hypothesis, this is equal to $\bigcup\left\{\llbracket \tau \rrbracket_{h}: h \in \mathcal{H}\right\} \cap \llbracket \xi \rrbracket_{g}=\bigcup\left\{\llbracket \tau \rrbracket_{h} \cap \llbracket \xi \rrbracket_{g}: h \in \mathcal{H}\right\}=\bigcup\left\{\llbracket \tau \rrbracket_{h} \cap \llbracket \xi \rrbracket_{h}: h \in \mathcal{H}\right\}=$ $\bigcup\left\{\llbracket \sigma \rrbracket_{h}: h \in \mathcal{H}\right\}$.

Finally, suppose that $\sigma=\mu X \tau$. By monotonicity it is plain that $\llbracket \sigma \rrbracket_{g} \supseteq \llbracket \sigma \rrbracket_{h}$ for each $h \in \mathcal{H}$, so we have $\llbracket \sigma \rrbracket_{g} \supseteq \bigcup\left\{\llbracket \sigma \rrbracket_{h}: h \in \mathcal{H}\right\}$. For the converse, we recall that

$$
\llbracket \sigma \rrbracket_{g}=\bigcap\left\{U \subseteq W: \llbracket \tau \rrbracket_{g_{X}^{U}} \subseteq U\right\}
$$

and

$$
\bigcup_{h \in \mathcal{H}} \llbracket \sigma \rrbracket_{h}=\bigcup_{h \in \mathcal{H}} \bigcap\left\{U \subseteq W: \llbracket \tau \rrbracket_{h_{X}^{U}} \subseteq U\right\} .
$$

Let $w \in W$ and suppose that $w \notin \bigcup_{h \in \mathcal{H}} \llbracket \sigma \rrbracket_{h}$. Then for each $h \in \mathcal{H}$ there exists $U_{h} \subseteq W$ such that $\llbracket \tau \rrbracket_{h_{X}^{U_{h}}} \subseteq U_{h}$ and $w \notin U_{h}$. Let $\mathcal{H}^{\prime}=\left\{h_{X}^{U_{h}}: h \in \mathcal{H}\right\}$ and $g^{\prime}=\bigcup \mathcal{H}^{\prime}$. Clearly, $\mathcal{H}^{\prime}$ is also $\mathcal{Q}$-variant. So by the induction hypothesis, we obtain $\llbracket \tau \rrbracket_{g^{\prime}}=\bigcup\left\{\llbracket \tau \rrbracket_{h^{\prime}}: h^{\prime} \in \mathcal{H}^{\prime}\right\}$. As $\llbracket \tau \rrbracket_{h_{X}^{U_{h}}} \subseteq U_{h}$ for each $h \in \mathcal{H}$, we have $\bigcup\left\{\llbracket \tau \rrbracket_{h^{\prime}}: h^{\prime} \in \mathcal{H}^{\prime}\right\} \subseteq \bigcup_{h \in \mathcal{H}} U_{h}=V$, say. But plainly, $g^{\prime}=g_{X}^{V}$. Thus, we obtained that $\llbracket \tau \rrbracket_{g_{\mathrm{X}}^{V}} \subseteq V$. Now $w \notin V$, as $w \notin U_{h}$ for each $h \in \mathcal{H}$. Thus, $w \notin \bigcap\left\{U \subseteq W: \llbracket \tau \rrbracket_{g_{X}^{U}} \subseteq U\right\}=\llbracket \sigma \rrbracket_{g}$.

A related theorem was proved using games in [13, proposition 5.5.4]. We will see that proposition 4.4 has consequences for standard translations of $\mathcal{Q}$-skeletons. In fact it is fundamental to what follows.

NOTATION 4.5 We will frequently be working with skeletons of the form

$$
\sigma\left(p_{1}, \ldots, p_{m}, q_{1}, \ldots, q_{n}\right)
$$

and the following notation will be repeatedly useful. We will write $N=$ $\{1, \ldots, n\}$. Fix pairwise distinct first-order variables $x, y_{1}, \ldots, y_{n}$. For $U \subseteq$ $V \subseteq N$, we will write

$$
\sigma_{U / V}\left(x, y_{i}, P_{1}, \ldots, P_{m}, Q_{j}: i \in U, j \in N \backslash V\right)
$$

for the FO+LFP-formula obtained from $S T_{x}(\sigma)$ by replacing every atomic subformula $Q_{k}(v)$ (where $k \in V$ and $v$ is a variable) by the formula

$$
\begin{cases}v=y_{k}, & \text { if } k \in U, \\ \perp, & \text { otherwise. }\end{cases}
$$

Note that $\sigma_{U / V}$ is a FO+LFP-formula, not a mu-formula.

\footnotetext{
${ }^{1}$ This assumption is not used here.
} 
COROLLARY 4.6 Let $\mathcal{Q}=\left\{q_{1}, \ldots, q_{n}\right\}$ and let $\sigma\left(p_{1}, \ldots, p_{m}, q_{1}, \ldots, q_{n}\right)$ be a $\mathcal{Q}$-skeleton sentence. Then $S T_{x}(\sigma)$ is logically equivalent to

$$
\sigma^{*}=\sigma_{\emptyset / N}\left(x, P_{1}, \ldots, P_{m}\right) \vee \bigvee_{1 \leq k \leq n} \exists y_{k}\left(\sigma_{\{k\} / N}\left(x, y_{k}, P_{1}, \ldots, P_{m}\right) \wedge Q_{k}\left(y_{k}\right)\right) .
$$

Proof. Let $\mathcal{F}=(W, R)$ be a frame, and take any assignment $g$ into $\mathcal{F}$, and $a \in W$. It is enough to show that

$$
a \in \llbracket \sigma \rrbracket_{g} \Longleftrightarrow \mathcal{F}=\sigma^{*}\left(a, g\left(p_{1}\right), \ldots, g\left(p_{m}\right), g\left(q_{1}\right), \ldots, g\left(q_{n}\right)\right) .
$$

Let $\mathcal{H}$ be the set of all assignments $h$ into $\mathcal{F}$ such that for some $k \in N$ :

- $h\left(q_{k}\right) \subseteq g\left(q_{k}\right)$ and $\left|h\left(q_{k}\right)\right| \leq 1$,

- $h\left(q_{l}\right)=\emptyset$ for each $l \in N \backslash\{k\}$,

- $h(\xi)=g(\xi)$ for every $\xi \in(\mathcal{P} \cup \mathcal{V}) \backslash \mathcal{Q}$.

Note that $\mathcal{H} \neq \emptyset, \mathcal{H}$ is $\mathcal{Q}$-variant, and $\cup \mathcal{H}=g$. Now we prove (8). The right-hand side holds iff $\mathcal{F} \models \sigma_{\emptyset / N}\left(a, g\left(p_{1}\right), \ldots, g\left(p_{m}\right)\right)$ or there are $k \in N$ and $b \in g\left(q_{k}\right)$ with $\mathcal{F}=\sigma_{\{k\} / N}\left(a, b, g\left(p_{1}\right), \ldots, g\left(p_{m}\right)\right)$. By definition of $\sigma_{U / V}$ and $\mathcal{H}$, this is iff $a \in \llbracket \sigma \rrbracket_{h}$ for some $h \in \mathcal{H}$. By proposition 4.4, this is iff $a \in \llbracket \sigma \bigcup_{\mathcal{H}}=\llbracket \sigma \rrbracket_{g}$, as required.

Corollary 4.6 will be useful for PIA formulas, but to rewrite Sahlqvist formulas as we did in (1), we need to extend it to formulas that may not be $\left\{q_{1}, \ldots\right.$, $\left.q_{n}\right\}$-skeletons, but are only $\left\{q_{i}\right\}$-skeletons for each $i=1, \ldots, n$. Because of this weaker assumption, we have to settle for a more complicated conclusion, but the family resemblance should be clear.

COROLLARY 4.7 Suppose that $\sigma\left(p_{1}, \ldots, p_{m}, q_{1}, \ldots, q_{n}\right)$ is a $\left\{q_{i}\right\}$-skeleton for each $i=1, \ldots, n$. Then $S T_{x}(\sigma)$ is logically equivalent to

$$
\sigma^{N}=\exists y_{1} \ldots y_{n} \bigvee_{U \subseteq N}\left(\sigma_{U / N}\left(x, y_{1}, \ldots, y_{n}, P_{1}, \ldots, P_{m}\right) \wedge \bigwedge_{k \in U} Q_{k}\left(y_{k}\right)\right)
$$

We remark that if $\sigma$ is normal in $q_{k}$ - that is, $\sigma\left(\perp / q_{k}\right) \equiv \perp-$ then all disjuncts with $k \notin U$ are equivalent to $\perp$ and can be deleted.

Proof. The proof is by induction on $n$. The case $n=0$ is trivially true, since then, $\sigma^{N}=\sigma_{\emptyset / \emptyset}=S T_{x}(\sigma)$. Let $n>0$ and assume the result for $n-1$. Treating $Q_{n}$ as a $P$ and applying the inductive hypothesis to the atoms $q_{1}, \ldots, q_{n-1}$, with $N^{\prime}=\{1, \ldots, n-1\}$, shows that $S T_{x}(\sigma)$ is equivalent to

$$
\sigma^{N^{\prime}}=\exists y_{1} \ldots y_{n-1} \bigvee_{U \subseteq N^{\prime}}\left(\sigma_{U / N^{\prime}}\left(x, y_{1}, \ldots, y_{n-1}, \bar{P}, Q_{n}\right) \wedge \bigwedge_{k \in U} Q_{k}\left(y_{k}\right)\right),
$$


where we write $\bar{P}$ for $\left(P_{1}, \ldots, P_{m}\right)$. As $\sigma$ is a $\left\{q_{n}\right\}$-skeleton, corollary 4.6 tells us that $S T_{x}(\sigma)$ is also equivalent to

$$
\sigma_{\emptyset /\{n\}}\left(x, \bar{P}, Q_{1}, \ldots, Q_{n-1}\right) \vee \exists y_{n}\left(\sigma_{\{n\} /\{n\}}\left(x, y_{n}, \bar{P}, Q_{1}, \ldots, Q_{n-1}\right) \wedge Q_{n}\left(y_{n}\right)\right) .
$$

Using (9) and the definitions of $\sigma_{\emptyset /\{n\}}$ and $\sigma_{\{n\} /\{n\}}$, the first disjunct of this is equivalent to

$$
\exists y_{1} \ldots y_{n-1} \bigvee_{\substack{U \subseteq N \\ n \notin U}}\left(\sigma_{U / N}\left(x, y_{1}, \ldots, y_{n-1}, \bar{P}\right) \wedge \bigwedge_{k \in U} Q_{k}\left(y_{k}\right)\right),
$$

and the second to

$$
\exists y_{1} \ldots y_{n} \bigvee_{\substack{U \subseteq N \\ n \in U}}\left(\sigma_{U / N}\left(x, y_{1}, \ldots, y_{n}, \bar{P}\right) \wedge \bigwedge_{k \in U} Q_{k}\left(y_{k}\right)\right) .
$$

$S T_{x}(\sigma)$ is equivalent to the disjunction of these, and so to $\sigma^{N}$, which completes the induction.

\subsection{Skeletons and PIA formulas}

In this section we will prove that any PIA sentence has a standard translation equivalent to a conjunction of 'genuine' PIA (positive implies atomic) formulas of $\mathrm{FO}+\mathrm{LFP}$.

DEFINITION 4.8 Let $\mathcal{Q} \subseteq \mathcal{P}$ and let $\sigma$ be a $\mathcal{Q}$-skeleton.

1. $\sigma$ is said to be normal if the formula obtained by replacing every free occurrence of every $\xi \in \mathcal{Q} \cup \mathcal{V}$ in $\sigma$ by $\perp$ is logically equivalent to $\perp$. ( $\mathcal{Q}$ is understood tacitly here. Atoms in $\mathcal{P} \backslash \mathcal{Q}$ are not altered in $\sigma$.)

2. We write $\sigma^{\mathcal{Q}}$ (the 'dual' of $\sigma$ ) for the formula obtained from $\neg \sigma$ by replacing each free occurrence of each $\xi \in \mathcal{Q} \cup \mathcal{V}$ by $\neg \xi$. Atoms in $\mathcal{P} \backslash \mathcal{Q}$ are unchanged and hence become negative in $\sigma^{\mathcal{Q}}$.

The following is as we would expect when taking duals.

LEMMA 4.9 Let $\sigma, \sigma_{1}, \sigma_{2}$ be $\mathcal{Q}$-skeletons. Then

1. $\left(\sigma_{1} \vee \sigma_{2}\right)^{\mathcal{Q}} \equiv \sigma_{1}^{\mathcal{Q}} \wedge \sigma_{2}^{\mathcal{Q}}$,

2. $(\diamond \sigma)^{\mathcal{Q}} \equiv \square \sigma^{\mathcal{Q}}$,

3. $(\mu X \sigma)^{\mathcal{Q}} \equiv \nu X \sigma^{\mathcal{Q}}$.

Proof. We prove only the last case. Let $\sigma(\bar{p}, \bar{q}, X, \bar{Y})$ be given, where $\bar{p}$ are atoms not in $\mathcal{Q}, \bar{q}$ are atoms in $\mathcal{Q}$, and $X, \bar{Y}$ are fixed point variables. Then in the obvious notation, $(\mu X \sigma)^{\mathcal{Q}}=\neg \mu X \sigma(\bar{p}, \neg \bar{q}, X, \neg \bar{Y}) \equiv \nu X \neg \sigma(\bar{p}, \neg \bar{q}, \neg X, \neg \bar{Y})=$ $\nu X \sigma^{\mathcal{Q}}$. 
This gives us the following alternative view of PIA formulas. In the lemma, formulas may have free fixed point variables but we do not display them.

LEMMA 4.10 Let $s_{1}, \ldots, s_{n}, q_{1}, \ldots, q_{n} \in \mathcal{P}$ be pairwise distinct atoms, and $\mathcal{Q}=\left\{q_{1}, \ldots, q_{n}\right\}$. For a mu-formula $\varphi$, let $\varphi^{*}=\varphi\left(s_{1} / q_{1}, \ldots, s_{n} / q_{n}\right)$ be the result of simultaneously replacing every atomic subformula $q_{i}$ of $\varphi$ by $s_{i}(1 \leq$ $i \leq n)$. Let $\beta\left(s_{1}, \ldots, s_{n}\right)$ be a PIA formula. Then $\beta \equiv\left(\sigma^{\mathcal{Q}}\right)^{*}$ for some normal $\mathcal{Q}$-skeleton $\sigma\left(q_{1}, \ldots, q_{n}, s_{1}, \ldots, s_{n}\right)$.

Proof. By induction on $\beta$. If $\beta$ is an atom $s_{i}$, we have $s_{i}=\left(\sigma^{\mathcal{Q}}\right)^{*}$ where $\sigma=q_{i}$ (a normal $\mathcal{Q}$-skeleton). if $\beta$ is a fixed point variable $X$, then $X=\left(\sigma^{\mathcal{Q}}\right)^{*}$ where $\sigma=X$ (again, $X$ is a normal $\mathcal{Q}$-skeleton). Suppose that $\beta_{1} \equiv\left(\sigma_{1}^{\mathcal{Q}}\right)^{*}$ and $\beta_{2} \equiv\left(\sigma_{2}^{\mathcal{Q}}\right)^{*}$, for normal $\mathcal{Q}$-skeletons $\sigma_{1}, \sigma_{2}$.

- Let $\sigma=\sigma_{1} \vee \sigma_{2}$ - plainly a normal $\mathcal{Q}$-skeleton. By lemma $4.9, \beta_{1} \wedge \beta_{2} \equiv$ $\left(\sigma_{1}^{\mathcal{Q}}\right)^{*} \wedge\left(\sigma_{2}^{\mathcal{Q}}\right)^{*} \equiv\left(\sigma_{1}^{\mathcal{Q}} \wedge \sigma_{2}^{\mathcal{Q}}\right)^{*} \equiv\left(\left(\sigma_{1} \vee \sigma_{2}\right)^{\mathcal{Q}}\right)^{*}=\left(\sigma^{\mathcal{Q}}\right)^{*}$

- By lemma $4.9, \square \beta_{1} \equiv \square\left(\sigma_{1}^{\mathcal{Q}}\right)^{*} \equiv\left(\left(\diamond \sigma_{1}\right)^{\mathcal{Q}}\right)^{*}$, and $\diamond \sigma_{1}$ is normal.

- For a fixed point variable $X$, let $\sigma$ be the $\mathcal{Q}$-skeleton $\mu X \sigma_{1}$. It is clearly normal. By lemma 4.9, $\nu X \beta_{1} \equiv \nu X\left(\sigma_{1}^{\mathcal{Q}}\right)^{*} \equiv\left(\left(\mu X \sigma_{1}\right)^{\mathcal{Q}}\right)^{*}=\left(\sigma^{\mathcal{Q}}\right)^{*}$.

- Finally we tackle the case $\pi \rightarrow \beta_{1}$ where $\pi\left(s_{1}, \ldots, s_{n}\right)$ is a positive modal mu-sentence. Now $\pi$ involves no atoms from $\mathcal{Q}$. So $\sigma=\pi \wedge \sigma_{1}$ is a normal $\mathcal{Q}$-skeleton, and $\pi \rightarrow \beta_{1} \equiv \pi \rightarrow\left(\sigma_{1}^{\mathcal{Q}}\right)^{*}=\left(\pi \rightarrow \sigma_{1}^{\mathcal{Q}}\right)^{*} \equiv\left(\neg\left(\pi \wedge \neg \sigma_{1}^{\mathcal{Q}}\right)\right)^{*} \equiv$ $\left(\left(\pi \wedge \sigma_{1}\right)^{\mathcal{Q}}\right)^{*}=\left(\sigma^{\mathcal{Q}}\right)^{*}$.

This completes the induction and the proof.

COROLLARY 4.11 Let $\beta\left(s_{1}, \ldots, s_{n}\right)$ be a PIA sentence. Then $S T_{x}(\beta)$ is equivalent to a 'PIA system' of $F O+L F P$ of the form

$$
\bigwedge_{1 \leq k \leq n} \forall y_{k}\left(\xi_{i}\left(x, y_{k}, S_{1}, \ldots, S_{n}\right) \rightarrow S_{k}\left(y_{k}\right)\right)
$$

where each $\xi_{k}$ is positive in $S_{1}, \ldots, S_{n}$.

Proof. Write $\bar{S}$ for $S_{1}, \ldots, S_{n}$. By lemma 4.10 we have $\beta \equiv\left(\sigma^{\mathcal{Q}}\right)^{*}$ for some normal $\mathcal{Q}$-skeleton sentence $\sigma\left(q_{1}, \ldots, q_{n}, s_{1}, \ldots, s_{n}\right)$. By corollary 4.6,

$$
S T_{x}(\sigma) \equiv \bigvee_{1 \leq k \leq n} \exists y_{k}\left(\sigma_{\{k\} / N}\left(x, y_{k}, \bar{S}\right) \wedge Q_{k}\left(y_{k}\right)\right)
$$

(By normality, the disjunct $\sigma_{\emptyset / N}$ in the corollary is equivalent to $\perp$ and we can dispense with it.) So, extending $-{ }^{*}$ to standard translations in the obvious way,

$$
\begin{aligned}
S T_{x}(\beta) \equiv S T_{x}\left(\left(\sigma^{\mathcal{Q}}\right)^{*}\right) & \equiv\left(\neg \bigvee_{1 \leq k \leq n} \exists y_{k}\left(\sigma_{\{k\} / N}\left(x, y_{k}, \bar{S}\right) \wedge \neg Q_{k}\left(y_{k}\right)\right)\right)^{*} \\
& \equiv \neg \bigvee_{1 \leq k \leq n} \exists y_{k}\left(\sigma_{\{k\} / N}\left(x, y_{k}, \bar{S}\right) \wedge \neg S_{k}\left(y_{k}\right)\right)
\end{aligned}
$$




$$
\equiv \bigwedge_{1 \leq k \leq n} \forall y_{k}\left(\sigma_{\{k\} / N}\left(x, y_{k}, \bar{S}\right) \rightarrow S_{k}\left(y_{k}\right)\right)
$$

which is in the required form.

We conclude that the standard translation of a PIA sentence $\beta\left(s_{1}, \ldots, s_{n}\right)$ is equivalent to a conjunction of FO+LFP-formulas in PIA form, one for each atom $s_{1}, \ldots, s_{n}$, but whose positive antecedents potentially involve all of $S_{1}, \ldots$, $S_{n}$. We will be able to compute a minimal assignment as in the usual PIA case, but using simultaneous fixed points.

\subsection{Skeletons and Sahlqvist formulas}

The definition of Sahlqvist formula is chosen so that we can view Sahlqvist formulas in terms of skeletons, by the following analogue of lemma 4.10.

LEMMA 4.12 Let $\varphi$ be a Sahlqvist formula whose free variables are among $X_{1}, \ldots, X_{t}$. Then there are a formula $\sigma\left(p_{1}, \ldots, p_{m}, q_{1}, \ldots, q_{n}, X_{1}, \ldots, X_{t}\right)$ that is a $\left\{q_{i}\right\}$-skeleton for each $i=1, \ldots, n$, negative sentences $\gamma_{1}, \ldots, \gamma_{m}$, and PIA sentences $\beta_{1}, \ldots, \beta_{n}$ (not necessarily distinct), such that

$$
\varphi \equiv \neg \sigma\left(\gamma_{1} / p_{1}, \ldots, \gamma_{m} / p_{m}, \beta_{1} / q_{1}, \ldots, \beta_{n} / q_{n}, \neg X_{1} / X_{1}, \ldots, \neg X_{t} / X_{t}\right) .
$$

Proof. By induction on $\varphi$. If $\varphi$ is a positive sentence then $\varphi \equiv \neg \sigma(\neg \varphi / p)$ where $\sigma=p$. If $\varphi$ is a negated PIA sentence $\neg \beta$ then $\varphi \equiv \neg \sigma(\beta / q)$ where $\sigma=q$. If $\varphi$ is a fixed point variable $X$, then $\varphi \equiv \neg \sigma(\neg X / X)$ for $\sigma=X$. Assume (11); then (11) holds with $\varphi$ replaced by $\square \varphi$ and $\sigma$ by $\diamond \sigma$. Also, taking $\nu X_{1}$ as an example,

$$
\begin{aligned}
& \nu X_{1} \varphi \\
\equiv & \nu X_{1} \neg \sigma\left(\gamma_{1} / p_{1}, \ldots, \gamma_{m} / p_{m}, \beta_{1} / q_{1}, \ldots, \beta_{n} / q_{n}, \neg X_{1} / X_{1}, \ldots, \neg X_{t} / X_{t}\right) \\
\equiv & \neg \mu X_{1} \sigma\left(\gamma_{1} / p_{1}, \ldots, \beta_{n} / q_{n}, X_{1}, \neg X_{2} / X_{2}, \ldots, \neg X_{t} / X_{t}\right),
\end{aligned}
$$

which is of the form (11).

Suppose in the obvious notation that

$$
\varphi \equiv \neg \sigma(\bar{\gamma} / \bar{p}, \bar{\beta} / \bar{q}, \neg \bar{X} / \bar{X}), \quad \varphi^{\prime} \equiv \neg \sigma^{\prime}\left(\bar{\gamma}^{\prime} / \bar{p}^{\prime}, \bar{\beta}^{\prime} / \bar{q}^{\prime}, \neg \bar{X}^{\prime} / \bar{X}^{\prime}\right),
$$

where $\sigma(\bar{p}, \bar{q}, \bar{X})$ is a $\{q\}$-skeleton for every $q$ in $\bar{q}$, and $\sigma^{\prime}\left(\bar{p}^{\prime}, \bar{q}^{\prime}, \bar{X}^{\prime}\right)$ is a $\left\{q^{\prime}\right\}$ skeleton for every $q^{\prime}$ in $\bar{q}^{\prime}$. We can suppose without loss of generality that no atom in $\bar{q}$ occurs in $\sigma^{\prime}$ and no atom in $\bar{q}^{\prime}$ occurs in $\sigma$. By remark 4.2, $\sigma, \sigma^{\prime}$, and hence $\sigma \vee \sigma^{\prime}$ are $\{q\}$-skeletons and $\left\{q^{\prime}\right\}$-skeletons for every $q$ in $\bar{q}$ and $q^{\prime}$ in $\bar{q}^{\prime}$, and clearly, $\varphi \wedge \varphi^{\prime} \equiv \neg\left(\sigma \vee \sigma^{\prime}\right)\left(\bar{\gamma} / \bar{p}, \bar{\gamma}^{\prime} / \bar{p}^{\prime}, \bar{\beta} / \bar{q}, \bar{\beta}^{\prime} / \bar{q}^{\prime}, \neg \bar{X} / \bar{X}, \neg \bar{X}^{\prime} / \bar{X}^{\prime}\right)$ as required. This covers the case $\varphi \wedge \varphi^{\prime}$.

Now suppose that $\varphi \vee \varphi^{\prime}$ is a Sahlqvist formula. Certainly, $\varphi \vee \varphi^{\prime} \equiv$ $\neg\left(\sigma \wedge \sigma^{\prime}\right)\left(\bar{\gamma} / \bar{p}, \bar{\gamma}^{\prime} / \bar{p}^{\prime}, \bar{\beta} / \bar{q}, \bar{\beta}^{\prime} / \bar{q}^{\prime}, \neg \bar{X} / \bar{X}, \neg \bar{X}^{\prime} / \bar{X}^{\prime}\right)$. But we need to check that $\left(\sigma \wedge \sigma^{\prime}\right)\left(\bar{p} \bar{p}^{\prime}, \bar{q} \bar{q}^{\prime}, \bar{X} \bar{X}^{\prime}\right)$ is a $\{\xi\}$-skeleton for each atom $\xi$ in $\bar{q} \bar{q}^{\prime}$. 
If $\varphi, \varphi^{\prime}$ are both sentences, then we can suppose that $\sigma, \sigma^{\prime}$ are too. For each atom $q$ in $\bar{q}$ (resp., $q^{\prime}$ in $\bar{q}^{\prime}$ ), it is plain that $\sigma^{\prime}$ (resp. $\sigma$ ) is a positive sentence not involving it. So $\sigma \wedge \sigma^{\prime}$ is a $\{\xi\}$-skeleton for each $\xi$ in $\bar{q} \bar{q}^{\prime}$.

Suppose instead that $\varphi$ is not a sentence (the other case is similar). Then (see definition 3.4) $\varphi^{\prime}$ is a positive sentence and consequently we may assume that $\sigma^{\prime}=p$ (as in the base case above) and $\bar{q}^{\prime}$ is empty. Now for each $q$ in $\bar{q}, \sigma^{\prime}$ is a positive sentence not involving $q$, so $\left(\sigma \wedge \sigma^{\prime}\right)\left(\bar{p} \bar{p}^{\prime}, \bar{q}, \bar{X} \bar{X}^{\prime}\right)$ is a $\{q\}$-skeleton. This completes the proof.

\subsection{Sahlqvist correspondence for mu-calculus}

We are now ready to prove our main result.

THEOREM 4.13 Any Sahlqvist mu-sentence $\varphi\left(s_{1}, \ldots, s_{t}\right)$ has a FO+LFP frame correspondent - a sentence $\chi_{\varphi}$ of $F O+L F P$ with the property that for any frame $\mathcal{F}$, we have $\mathcal{F} \models \chi_{\varphi}$ iff $\varphi$ is valid in $\mathcal{F}$. The correspondent $\chi_{\varphi}$ can be computed from $\varphi$ by an algorithm.

Proof. We follow the same steps as in our original account in section 3. Let $\mathcal{F}=(W, R)$ be any Kripke frame.

Step 1. Assume that $\varphi$ is not valid in $\mathcal{F}$. This is the case iff there are an assignment $h$ into $\mathcal{F}$ and $a \in W$ with $(\mathcal{F}, h), a \models \neg \varphi$. Now by lemma 4.12,

$$
\neg \varphi \equiv \sigma\left(\gamma_{1} / p_{1}, \ldots, \gamma_{m} / p_{m}, \beta_{1} / q_{1}, \ldots, \beta_{n} / q_{n}\right),
$$

where $\sigma\left(p_{1}, \ldots, p_{m}, q_{1}, \ldots, q_{n}\right)$ is a sentence that is a $\left\{q_{i}\right\}$-skeleton for each $1 \leq$ $i \leq n$, and $\gamma_{1}, \ldots, \gamma_{m}$ are negative sentences and $\beta_{1}, \ldots, \beta_{n}$ PIA sentences written with the atoms $s_{1}, \ldots, s_{t}$. So

$$
(\mathcal{F}, h), a \models \sigma\left(\gamma_{1} / p_{1}, \ldots, \gamma_{m} / p_{m}, \beta_{1} / q_{1}, \ldots, \beta_{n} / q_{n}\right) .
$$

Step 2. By corollary $4.7, S T_{x}\left(\sigma\left(p_{1}, \ldots, p_{m}, q_{1}, \ldots, q_{n}\right)\right)$ is logically equivalent to

$$
\exists y_{1} \ldots y_{n} \bigvee_{U \subseteq N}\left(\sigma_{U / N}\left(x, P_{1}, \ldots, P_{m}, y_{1}, \ldots, y_{n}\right) \wedge \bigwedge_{k \in U} Q_{k}\left(y_{k}\right)\right)
$$

So by (12), we see that $\varphi$ is not valid in $\mathcal{F}$ iff there are an assignment $h$ into $\mathcal{F}$, $a, b_{1}, \ldots, b_{n} \in W$, and $U \subseteq N=\{1, \ldots, n\}$ with

$$
\mathcal{F} \models \sigma_{U / N}\left(a, \llbracket \gamma_{1} \rrbracket_{h}, \ldots, \llbracket \gamma_{m} \rrbracket_{h}, b_{1}, \ldots, b_{n}\right) \text { and } \bigwedge_{k \in U}\left(b_{k} \in \llbracket \beta_{k} \rrbracket_{h}\right) .
$$

Step 3. We now plan to replace $h$ by a 'minimal' assignment $h^{\circ}$, preserving (13). This assignment will depend uniformly on $b_{1}, \ldots, b_{n}$, as before, and it will also depend on $U$. 
Each $\beta_{k}\left(s_{1}, \ldots, s_{t}\right)(1 \leq k \leq n)$ is PIA, so by corollary 4.11 its standard translation $S T_{y_{k}}\left(\beta_{k}\right)$ is equivalent to a FO+LFP-formula of the form in (10):

$$
\bigwedge_{1 \leq l \leq t} \forall z_{l}\left(\psi_{l}^{k}\left(y_{k}, z_{l}, \bar{S}\right) \rightarrow S_{l}\left(z_{l}\right)\right)
$$

where we write $\bar{S}$ for ' $S_{1}, \ldots, S_{t}$ ', and each $\psi_{l}^{k}$ is positive in $S_{1}, \ldots, S_{t}$. So the last part of (13) says precisely that

$$
\left(\mathcal{F}, h\left(s_{1}\right), \ldots, h\left(s_{t}\right)\right) \models \forall z_{l}\left(\psi_{l}^{k}\left(b_{k}, z_{l}, \bar{S}\right) \rightarrow S_{l}\left(z_{l}\right)\right)
$$

for each $l=1, \ldots, t$ and each $k \in U$. This condition is plainly equivalent to $\left(\mathcal{F}, h\left(s_{1}\right), \ldots, h\left(s_{t}\right)\right) \models \bigwedge_{k \in U} \forall z_{l}\left(\psi_{l}^{k}\left(b_{k}, z_{l}, \bar{S}\right) \rightarrow S_{l}\left(z_{l}\right)\right)$ for each $1 \leq l \leq t$, and so to:

$$
\begin{aligned}
\left(\mathcal{F}, h\left(s_{1}\right), \ldots, h\left(s_{t}\right)\right) \models \forall z_{l}\left(\rho_{l}^{U}\left(z_{l}, b_{1}, \ldots, b_{n}, \bar{S}\right) \rightarrow\right. & \left.S_{l}\left(z_{l}\right)\right) \\
& \text { for each } 1 \leq l \leq t,
\end{aligned}
$$

where

$$
\rho_{l}^{U}\left(z_{l}, y_{1}, \ldots, y_{n}, \bar{S}\right)=\bigvee_{k \in U} \psi_{l}^{k}\left(y_{k}, z_{l}, \bar{S}\right) .
$$

Now each $\rho_{l}^{U}$ is positive in $S_{1}, \ldots, S_{t}$. So (16) is in 'simultaneous PIA' form, and a minimal assignment to each $s_{l}$ exists. Call this assignment $h^{\circ}$. As we said, it depends on $b_{1}, \ldots, b_{n}$, and $U$ (this is not explicit in the notation $h^{\circ}$ ). For $s \in \mathcal{P} \backslash\left\{s_{1}, \ldots, s_{t}\right\}$ we have $h^{\circ}(s)=\emptyset$.

If we replace $h$ by $h^{\circ}$ in (13), the condition $b_{k} \in \llbracket \beta_{k} \rrbracket_{h^{\circ}}$ for each $k \in U$ is automatic $-h^{\circ}$ is by definition the minimal assignment that ensures this. Moreover, $h^{\circ}(s) \subseteq h(s)$ for all atoms $s$. By antitonicity, $\llbracket \gamma_{l} \rrbracket_{h} \subseteq \llbracket \gamma_{l} \rrbracket_{h^{\circ}}$ for each $1 \leq l \leq m$. As $\sigma_{U / N}$ is positive in $P_{1}, \ldots, P_{m}$, we have $\mathcal{F} \models \sigma_{U / N}\left(a, \llbracket \gamma_{1} \rrbracket_{h^{\circ}}, \ldots, \llbracket \gamma_{m} \rrbracket_{h^{\circ}}\right.$, $\left.b_{1}, \ldots, b_{n}\right)$.

We conclude from (13) that $\varphi$ is not valid in $\mathcal{F}$ iff there are $a, b_{1}, \ldots, b_{n} \in W$, and $U \subseteq N$ such that with the above $h^{\circ}$,

$$
\mathcal{F} \models \sigma_{U / N}\left(a, \llbracket \gamma_{1} \rrbracket_{h^{\circ}}, \ldots, \llbracket \gamma_{m} \rrbracket_{h^{\circ}}, b_{1}, \ldots, b_{n}\right) .
$$

Step 4. Moreover, the minimal assignment $h^{\circ}$ satisfying (16) is definable in FO+LFP: for each atom $s_{l}(1 \leq l \leq t), h^{\circ}\left(s_{l}\right)$ is the set of all $c \in W$ that satisfy the FO+LFP-formula $\eta_{l}^{U}\left(c, b_{1}, \ldots, b_{n}\right)$, where

$$
\eta_{l}^{U}\left(z_{l}, y_{1}, \ldots, y_{n}\right)=\left[S-L F P\left(l, z_{1}, S_{1}, \ldots, z_{t}, S_{t}\right) \rho_{1}^{U}, \ldots, \rho_{t}^{U}\right]\left(z_{l}, y_{1}, \ldots, y_{n}\right) .
$$

See $\S 2.2$ for ' $S$ - $L F P$ '. The $\eta_{l}^{U}$ are well formed since the $\rho_{l}^{U}$ from (17) are positive in $S_{1}, \ldots, S_{t}$. 
Summing up. Let $\omega_{U}\left(x, y_{1}, \ldots, y_{n}\right)$ be the formula obtained as follows. We take $\sigma_{U / N}\left(x, P_{1}, \ldots, P_{m}, y_{1}, \ldots, y_{n}\right)$ and replace each atomic subformula $P_{j}(v)$ ( $1 \leq j \leq m, v$ a variable) by the formula obtained from $S T_{v}\left(\gamma_{j}\right)$ by replacing each atomic subformula $S_{l}(z)$ (for some $1 \leq l \leq t$ and variable $z$ ) by $\eta_{l}^{U}\left(z / z_{l}, y_{1}, \ldots, y_{n}\right)$ from (19) (the parts of $\eta_{l}^{U}$ are given in (17) and (14)). Then (18) is equivalent to $\mathcal{F} \models \omega_{U}\left(a, b_{1}, \ldots, b_{n}\right)$, and $\varphi$ is not valid in $\mathcal{F}$ iff there are $a, b_{1}, \ldots, b_{n} \in W$ and $U \subseteq N$ such that this holds. We conclude that the original statement that $\varphi$ is not valid in $\mathcal{F}$ is equivalent to

$$
\mathcal{F} \models \exists x y_{1} \ldots y_{n} \bigvee_{U \subseteq N} \omega_{U}\left(x, y_{1}, \ldots, y_{n}\right) .
$$

Thus we obtain our correspondent $\chi_{\varphi}$ as the negation of this.

\section{$5 \quad$ Examples}

We will now give a few examples concerning frame correspondents. We explained the algorithm that constructs the correspondents in full detail in section 4 , and in spirit in section 3. In the examples, we will take an informal approach true to the spirit of the algorithm. The reader may like to apply the algorithm to the examples following the precise steps of the preceding section. One more example will be given in footnote 2 below.

\section{$5.1 \quad$ Löb's formula, $\square(\square p \rightarrow p) \rightarrow \square p$}

We simply state the correspondence: $\mathcal{F}, x \models \square(\square p \rightarrow p) \rightarrow \square p$ iff (1) $R$ is transitive from $x$, and (2) $R$ is conversely well-founded at $x$. Note that the antecedent $\square(\square p \rightarrow p)$ is PIA, and we can see that its minimal valuation stated as a fixed-point by our general procedure amounts to the set $\left\{y: \forall z\left(R^{*} y z \rightarrow\right.\right.$ $R x z) \wedge$ no infinite sequence starts from $y\}$. Substituting this into the consequent gives the above frame-equivalent.

Now that we have PIA forms, we can go back to earlier work on non-firstorder correspondence and see what was going on. For instance, the modal axiom $(\diamond p \wedge \square(p \rightarrow \square p)) \rightarrow p$ discussed in [3] has a PIA conjunct $\square(p \rightarrow \square p)$ in its antecedent. Its corresponding frame property is easily determined.

\subsection{Axioms of propositional dynamic logic (PDL)}

Consider the axioms of PDL, treating complex program expressions as new relation symbols. For instance, the characteristic axiom for composition, $[a ; b] p \leftrightarrow$ $[a][b] p$, may be viewed as $[c] p \leftrightarrow[a][b] p$. This axiom consists of two implications that are clearly Sahlqvist forms. Computing their frame equivalents via the usual algorithm yields $R_{c}=R_{a} \circ R_{b}$, where $\circ$ is composition of binary relations. Now consider the two axioms for Kleene star: (i) $\left[a^{*}\right] p \rightarrow p \wedge[a]\left[a^{*}\right] p$, (ii) $p \wedge\left[a^{*}\right](p \rightarrow[a] p) \rightarrow\left[a^{*}\right] p$. These may be viewed as (i) $[b] p \rightarrow p \wedge[a][b] p$,

(ii) $p \wedge[b](p \rightarrow[a] p) \rightarrow[b] p$. Of these, the first is standard first-order Sahlqvist. 
What it says is that $I d \subseteq R_{b}$ and $R_{a} \circ R_{b} \subseteq R_{b}$. The second principle has an antecedent that is PIA by the rules of our syntax. Suppressing a precise calculation here, in conjunction with the preceding two inclusions it says that the relation $R_{b}$ is equal to the reflexive-transitive closure $R_{a}^{*}$.

\section{$5.3 \varphi_{1}=\square^{+} s \rightarrow s$}

Here, $\square^{+} s$ abbreviates $\nu X \square(s \wedge X)$, which defines the 'transitive closure' of $\square$. We could treat $\varphi_{1}$ as a classical Sahlqvist formula in a modal signature with the box $\square^{+}$with accessibility relation $R^{+}$, calculate its correspondent by the classical method (§3.1) as $\forall x R^{+}(x, x)$, and then replace $R^{+}(x, x)$ by its FO+LFP definition $[\operatorname{LFP}(Q, x, y) . R(x, y) \vee \exists z(R(x, z) \wedge Q(z, y))](x, x)$, or $\exists y(y=x \wedge$ $[L F P(P, x) . R(x, y) \vee \exists z(R(x, z) \wedge P(z))](x, y))$.

Alternatively, we can use our algorithm. Written out in the mu-calculus, $\varphi_{1}$ is $\nu X \square(s \wedge X) \rightarrow s$. It is valid in a frame $\mathcal{F}$ at a world $x$ iff $(\mathcal{F}, h), x \models$ $\nu X \square(s \wedge X) \rightarrow s$ for all assignments $h$ into $\mathcal{F}$.

Let $\mathcal{H}$ be the set of assignments $h$ (into $\mathcal{F}$ ) with $(\mathcal{F}, h), x=\nu X \square(s \wedge X)$. We will show that there is a 'smallest' $h^{\circ}$ (with minimum $h(s)$ ) in $\mathcal{H}$. Then $\varphi_{1}$ is valid in $\mathcal{F}$ iff $(\mathcal{F}, h), x=s$ for all $h \in \mathcal{H}$. Since $s$ is positive, this holds iff $\left(\mathcal{F}, h^{\circ}\right), x \models s$.

We calculate $h^{\circ}$ using PIA methods. Clearly, $\nu X \square(s \wedge X) \equiv[\neg \mu X \diamond(s \vee$ $X)](\neg s / s)$. As $\mu X \diamond(s \vee X)$ is normal and completely additive in $s$, its standard translation $S T_{x}$ at $x$ is equivalent to $\exists v(\lambda(v, x) \wedge S(v))$, where

$$
\lambda(v, x)=[L F P(X, x) . \exists y(R(x, y) \wedge(y=v \vee X(y)))](v, x) .
$$

So

$$
S T_{x}(\nu X \square(s \wedge X)) \equiv \forall v(\lambda(v, x) \rightarrow S(v)) .
$$

This is in PIA form. The minimal assignment to $s$ with respect to $x$ is given by $\operatorname{LFP}(S, v)$ applied to the antecedent $\lambda(v, x)$. This is equivalent to $\lambda(v, x)$, as $S$ does not occur free in $\lambda$. ((20) is 'CIA' - 'constant implies atomic'.)

So the 'minimal' $h^{\circ} \in \mathcal{H}$ is given by $h^{\circ}(s)=\{v \in \mathcal{F}: \mathcal{F}=\lambda(v, x)\}$, and $\varphi$ is valid in $\mathcal{F}$ at $x$ iff $\left(\mathcal{F}, h^{\circ}\right), x \models s$, iff $\mathcal{F} \models \lambda(x / v, x)$. Consequently, $\varphi_{1}$ is valid in a frame $\mathcal{F}$ iff $\mathcal{F} \models \forall x \lambda(x / v, x)$ : i.e.,

$$
\mathcal{F} \models \forall x([L F P(X, x) . \exists y(R(x, y) \wedge(y=x \vee X(y))](x)) .
$$

This is our frame correspondent.

$$
5.4 \varphi_{2}=s \rightarrow \nu X\left(\square\left(X \wedge \neg s^{\prime}\right) \vee\left(\diamond s \wedge \diamond s^{\prime}\right)\right)
$$

This can be checked to conform to definition 3.4, if we replace the initial ' $s \rightarrow$ ' by ' $\neg s \vee$ '. The skeleton associated with $\varphi_{1}$ above was just $p \wedge q$. For $\varphi_{2}$, the skeleton is nontrivial: $\varphi_{2}$ is equivalent to the Sahlqvist mu-formula

$$
\neg \sigma\left(\eta / p, s / q, s^{\prime} / q^{\prime}\right),
$$


where (clearly) $s, s^{\prime}$ are PIA formulas, $\gamma=\neg\left(\diamond s \wedge \diamond s^{\prime}\right)$ is negative, and

$$
\sigma\left(p, q, q^{\prime}\right)=q \wedge \mu X\left(p \wedge \diamond\left(q^{\prime} \vee X\right)\right)
$$

is a $\{q\}$-skeleton and a $\left\{q^{\prime}\right\}$-skeleton. (It is not a $\{p\}$-skeleton, because in $p \wedge \diamond\left(q^{\prime} \vee X\right)$, the right-hand conjunct is not a sentence but the left-hand one involves $p$. Nor is it a $\left\{q, q^{\prime}\right\}$-skeleton.) The second conjunct of $\sigma$ is equivalent to a strict form of $p U q^{\prime}$. So $\varphi \equiv s \rightarrow \neg\left(\left[\neg\left(\diamond s \wedge \diamond s^{\prime}\right)\right] U s^{\prime}\right)$.

We calculate the frame correspondent of $\varphi_{2}$. We will suppress some parentheses to aid readability. Note that $\sigma$ is normal in $q$ and $q^{\prime}$, so (as we mentioned between the statement and proof of corollary 4.7) $S T_{x}(\sigma)$ is equivalent to the rather simple formula

$$
\begin{aligned}
& \exists y y^{\prime}\left(x=y \wedge\left[L F P(X, x) . P x \wedge \exists z\left(R x z \wedge\left(z=y^{\prime} \vee X z\right)\right)\right]\right. \\
& \left.\wedge Q y \wedge Q^{\prime} y^{\prime}\right) .
\end{aligned}
$$

We now take $S T_{x}(\gamma)=\neg\left(\exists v(R x v \wedge S v) \wedge \exists v\left(R x v \wedge S^{\prime} v\right)\right)$ and replace references to $S, S^{\prime}$ by the minimal valuations for them, which are $\{y\},\left\{y^{\prime}\right\}$, respectively. We obtain $\neg\left(\exists v(R x v \wedge v=y) \wedge \exists v\left(R x v \wedge v=y^{\prime}\right)\right)$, which simplifies to $\neg\left(R x y \wedge R x y^{\prime}\right)$. This is substituted for $P x$ in (21) and the conjuncts $Q y, Q^{\prime} y^{\prime}$ are deleted since they will automatically be true under the minimal assignment. We obtain

$$
\exists y y^{\prime}\left(x=y \wedge\left[L F P(X, x) . \neg\left(R x y \wedge R x y^{\prime}\right) \wedge \exists z\left(R x z \wedge\left(z=y^{\prime} \vee X z\right)\right)\right]\right),
$$

and this holds at a world $x$ iff $\varphi_{2}$ is not valid at $x$. So our frame correspondent for $\varphi_{2}$ expresses the negation of the above for all $x$, which boils down to:

$$
\forall x y y^{\prime}\left(x=y \rightarrow G F P[X, x]\left[\forall z\left(R x z \rightarrow\left(z \neq y^{\prime} \wedge X z\right)\right) \vee\left(R x y \wedge R x y^{\prime}\right)\right]\right) .
$$

The correspondent plainly 'says' that for any path $x=x_{0} R x_{1} R \ldots R x_{n}=y$ in the frame, with $n>0$, there is $i$ with $0 \leq i<n$ such that $R x_{i} x$ and $R x_{i} y$.

This raises some interesting connections with PDL. We do not believe that there is any PDL formula without tests that is valid in the same frames as $\varphi_{2}$, but $\varphi_{2}$ is valid in the same frames as

$$
\varphi_{3}=p \wedge\left\langle(? q ; a)^{*}\right\rangle p^{\prime} \rightarrow\left\langle(? q ; a)^{*}\right\rangle\left(\diamond p \wedge \diamond p^{\prime}\right),
$$

where $q$ is a new atom and $a$ is a program with accessibility relation $R$. The idea is roughly that if $(\mathcal{F}, h), x \models p \wedge\left\langle(? q ; a)^{*}\right\rangle p^{\prime}$, then there is $y$ with $R^{*} x y$ at which $p^{\prime}$ holds, and a path from $x$ to $y$ along which $q$ holds. The minimal values of $p, p^{\prime}, q$ are now $x, y$, and the path, respectively. The consequent now states that some world $t$ on the path is $R$-related to worlds satisfying these minimal values of $p, p^{\prime}$ : i.e., Rtx and $R t y$.

In general, the minimal value of $q$ (the path) is not unique, and considering automorphisms shows that it is not going to be definable in terms of $x, y$ in any logic at all. So such PDL-formulas seem to be (possibly much) more powerful than Sahlqvist mu-formulas. On the other hand, Sahlqvist mu-formulas allow 
rather free use of fixed points, and in expressive power may go beyond even PDL-formulas with tests. Consider for example $\mu X \square X$. As is well known, this defines the well-founded part of any model. This property appears not to be definable in PDL. The exact relationship between the two formalisms is to be the object of further study.

\subsection{McKinsey's axiom: $\square \diamond p \rightarrow \diamond \square p$}

Of course, not every modal mu-formula, or even every modal formula, has a frame correspondent in FO+LFP. It was mentioned in [4] that McKinsey's axiom $\varphi=\square \diamond p \rightarrow \diamond \square p$ has no such correspondent and that this can be proved using the Löwenheim-Skolem property for LFP (joint work by van Benthem and Goranko).

Here, we give a little more detail of the proof. It is based on [2]; see also [1, theorem 21] and [16, theorem 2.2]. Note first that $\varphi$ is equivalent to $\diamond(\square p \vee \square \neg p)$. Let $\mathcal{F}$ be the frame whose set of worlds consists of three disjoint parts: a root $r$; the natural numbers; and the infinite sets $X$ of natural numbers. The accessibility relation $R$ of $\mathcal{F}$ relates $r$ to every $X, X$ to every member of $X$, and each natural number to itself; these are the only instances of $R$. It can be verified that $\varphi$ is valid in $\mathcal{F}$, because for any assignment of $p$ into $\mathcal{F}$, there must be an infinite set $X$ of natural numbers all having the same truth value for $p$, and $\square p \vee \square \neg p$ is consequently true at such an $X$. Hence $\diamond(\square p \vee \square \neg p)$ is true at the root. Truth of $\varphi$ at all other worlds of $\mathcal{F}$ is easy to check.

Suppose for contradiction that $\chi$ is a (global) frame correspondent of $\varphi$ in $\mathrm{FO}+\mathrm{LFP}$, so that $\mathcal{F} \models \chi$. It follows from the proof of the downward Löwenheim-Skolem property for FO+LFP in [19, theorem 2.4] that there is a countable elementary substructure $\mathcal{F}_{0} \preceq \mathcal{F}$ containing all the natural numbers and with $\mathcal{F}_{0}=\chi$, and so $\varphi$ is valid in $\mathcal{F}_{0}$. To see that this is impossible, enumerate the sets of natural numbers in $\mathcal{F}_{0}$ as $X_{0}, X_{1}, \ldots$, and select by induction distinct natural numbers $x_{0}, y_{0}, x_{1}, y_{1}, \ldots$ in such a way that $x_{n}, y_{n} \in X_{n}$ for each $n$ (this is possible because $X_{n}$ is infinite). Now assign $p$ to $\left\{x_{0}, x_{1}, \ldots\right\}$. Every set $X_{n}$ in $\mathcal{F}_{0}$ contains a point $\left(x_{n}\right)$ satisfying $p$ and a point $\left(y_{n}\right)$ satisfying $\neg p$, so $\square p \vee \square \neg p$ is false at every $X_{n}$. Hence, $\varphi$ is false at the root.

\section{Related work}

This paper has focused on one particular line in Sahlqvist-style frame correspondence for modal fixed-point logics, going back to earlier work of the first author. However, we are by no means the first to put this area on the map, and there are other systematic takes on modal principles like Löb's Axiom or the PDL Induction Axiom. Here are two important earlier approaches.

One approach, going back to [14], uses a second-order correspondence language with general techniques of Skolemization and quantifier elimination to deal with a large variety of modal axioms. Outcomes for concrete modal axioms may then simplify to pure first-order or FO+LFP because of special syntactic 
features of these axioms. This line of work was inspired by the search for generic automated theorem proving with a wide spectrum of modal logics, making the axioms an input parameter of the system through a translation algorithm SCAN. Another paper systematizing reductio methods in this line is [10]. An elegant extended approach using a recursive version of Ackermann's Lemma is found in [21]. The latter paper may well be the first source for modal correspondence theory into FO+LFP.

A second main line, and closer to the more purely modal approach in this paper, is the work by Goranko, Vakarelov, and others on extending the modal syntax for which systematic correspondence arguments can work. A key reference is [18]. The authors discuss the idea of correspondence from modal logic to FO+LFP, and identify a large class of 'regular modal formulas' that are proved to have correspondents in FO+LFP. This program and especially, its algorithmic aspects, has been taken further, e.g., in [9]. The paper presents a recursive extension of the algorithm SQEMA for translating a large class of modal formulas (including the mentioned regular ones) into a fragment of the hybrid modal mu-calculus, which itself translates into FO+LFP. A good general source for placing these results in context is [17].

There are many obvious questions about the relation between the present work and the results in the mentioned traditions. In particular, how does our generalized mu-calculus Sahlqvist syntax relate to the above regular modal formulas $^{2}$ and their extension to formulas having correspondents in hybrid mucalculi that are fragments of the full FO+LFP? Also, how does the algorithm presented in our text relate to the SQEMA algorithm with its recursive extension? Finally, how do our fixed-point logic oriented proofs relate to the second-order logic-based style of the first-mentioned tradition, and can one find a link with second-order quantifier elimination methods?

We cannot pursue these issues here for lack of space, but they suggest a natural follow-up project doing justice to all existing approaches. Putting ideas together should enable us to see better where we stand with modal correspondence theory for fixed-point languages.

\section{Conclusions and future work}

We conclude with a discussion of further possible directions for future work.

Strengthening the modal base. In this paper we consider only the basic modal language extended with fixed point operators. However, there is room for further expansions involving hybrid modal languages, or the Guarded Fragment with

\footnotetext{
${ }^{2}$ As an appetizer, consider the inductive formula $D_{1}=s_{1} \wedge \square\left(\diamond s_{1} \rightarrow \square s_{2}\right) \rightarrow \diamond \square s_{2}$ of $[18$, example 35], which is stated as being 'not a Sahlqvist formula, nor... tautologically reducible to one'. It is equivalent to the Sahlqvist mu-formula $\neg \beta \vee \pi$, where $\beta=s_{1} \wedge \square\left(\diamond s_{1} \rightarrow \square s_{2}\right)$ is PIA and $\pi=\diamond \square s_{2}$ is positive. Corollary 4.11 yields that $S T_{x} \beta \equiv \forall y_{1}\left(x=y_{1} \rightarrow S_{1} y_{1}\right) \wedge$ $\forall y_{2}\left(\exists u\left(R x u \wedge \exists v\left(R u v \wedge S_{1} v\right) \wedge R u y_{2}\right) \rightarrow S_{2} y_{2}\right)$. As in theorem 4.13, we obtain minimal assignment $S_{1} y_{1} \leftrightarrow y_{1}=x$ and (hence) $S_{2} y_{2} \leftrightarrow \exists u\left(R x u \wedge R u x \wedge R u y_{2}\right)$. Using this value of $S_{2}$ in $S T_{x} \pi$ yields our final correspondent of $D_{1}: \forall x \exists y(R x y \wedge \forall z(R y z \rightarrow \exists u(R x u \wedge R u x \wedge R u z)))$, as in [18].
} 
fixed point operators. Extensions of classical Sahlqvist correspondence to these languages have already been studied in, e.g., [8]. We think our approach can be generalized in the same way.

Fragments of the mu-calculus. One can also look into an opposite direction, at languages weaker than mu-calculus, and examine the consequences of the Sahlqvist correspondence developed in this paper. One obvious candidate is propositional dynamic logic (PDL), which has already played a large role in our examples. ${ }^{3}$

The fixed-point correspondence language. We now turn to the other end of our Sahlqvist correspondence: the logic FO+LFP. It is of course of interest to know how much power of this logic we are really using. In other words, in what subfragment of FO+LFP do the correspondents of Sahlqvist mu-formulas 'land'? For the classical Sahlqvist correspondence this question has been answered by Kracht [20, 7]. But for the modal mu-calculus this question is wide open. ${ }^{4}$

Proof-theoretic aspects. Semantic correspondence arguments can be formalized in axiomatic proof-theoretic calculi. What often suffices are weak fragments of full monadic second-order logic, or of the full fixed-point logic FO+LFP. We intend to study these proof-theoretic aspects of our new results in more detail. Of special interest here is the greater deductive power of the $\mu$-calculus as such. For instance, it can prove in purely modal syntax that Löb's Axiom is equivalent to well-foundedness ( $\mu p \square p$ ) plus the K4-axiom $\square \varphi \rightarrow \square \square \varphi$. Thus, a richer modal logic formalizes correspondence facts about a poorer one.

Further questions. Of course one could also ask for analogues for the mucalculus of other famous definability results, such as the Goldblatt-Thomason theorem, which gives necessary and sufficient condition for a class of frames to be modally definable. Another example is Fine's theorem, which states that every elementarily definable modal logic is canonical. There are different ways to formulate canonicity for modal mu-logics, and a useful framework for this might be the admissible semantics of modal mu-calculus used in [6].

To sum everything up, we hope to have shown that the mu-calculus provides a natural new take on many traditional issues in modal definability, and that there is a lot of interesting syntactic and semantic structure awaiting further exploration.

Acknowledgement We thank the referee for very useful comments that greatly helped to improve the paper.

\footnotetext{
${ }^{3}$ Fontaine $[13, \S 5.5]$ characterizes PDL-formulas (with the restriction that these formulas may contain only one atom) as a certain subfragment of the mu-calculus.

${ }^{4} \mathrm{Mu}$-calculus formulas retain all the bisimulation-induced key semantic properties of modal ones, such as preservation under generated subframes, p-morphic images, disjoint unions. Can we find some further syntax restrictions?
} 


\section{References}

[1] P. Balbiani, V. Shehtman, and I. Shapirovsky, Every world can see a Sahlqvist world, Proc. Advances in Modal Logic (G. Governatori, I. Hodkinson, and Y. Venema, eds.), College Publications, 2006, pp. 69-85.

[2] J. van Benthem, A note on modal formulas and relational properties, Journal of Symbolic Logic 40 (1975), no. 1, 55-58.

[3] _ Modal logic and classical logic, Bibliopolis, Naples, 1985.

[4] _ Minimal predicates, fixed-points, and definability, J. Symbolic Logic 70 (2005), 696-712.

[5] _ Modal frame correspondences and fixed-points, Studia Logica 83 (2006), 133-155.

[6] N. Bezhanishvili and I. Hodkinson, Sahlqvist theorem for modal fixed point logic, (2010), submitted.

[7] P. Blackburn, M. de Rijke, and Y. Venema, Modal logic, Tracts in Theoretical Computer Science, Cambridge University Press, Cambridge, UK, 2001.

[8] B. ten Cate, M. Marx, and P. Viana, Hybrid logics with Sahlqvist axioms, Logic J. IGPL 13 (2005), 293-300.

[9] W. Conradie, V. Goranko, and D. Vakarelov, Algorithmic correspondence and completeness in modal logic $V$ : recursive extensions of SQEMA, J. Applied Logic 8 (2010), 319-333.

[10] P. Doherty, W. Łukaszewicz, and A. Szałas, Computing circumscription revisited: A reduction algorithm, J. Automated Reasoning 18 (1997), 297336.

[11] H-D. Ebbinghaus and J. Flum, Finite model theory, 2nd ed., Perspectives in mathematical logic, Springer-Verlag, New York, 1999.

[12] L. L. Esakia, Topological Kripke models, Soviet Math. Dokl. 15 (1974), $147-151$.

[13] G. Fontaine, Modal fixpoint logic: some model theoretic questions, Ph.D. thesis, ILLC, Amsterdam, 2010, ILLC Dissertation Series DS-2010-09.

[14] D. M. Gabbay and H-J. Ohlbach, Quantifier elimination in second-order predicate logic, Principles of Knowledge Representation and Reasoning (KR92) (B. Nebel, C. Rich, and W. Swartout, eds.), Morgan Kaufmann, 1992, pp. 425-435.

[15] S. Givant and Y. Venema, The preservation of Sahlqvist equations in completions of Boolean algebras with operators, Algebra Universalis 41 (1999), $47-84$. 
[16] R. Goldblatt and I. Hodkinson, The McKinsey-Lemmon logic is barely canonical, Australasian J. Logic 5 (2007), 1-19.

[17] V. Goranko and M. Otto, Model theory of modal logic, Handbook of Modal Logic (P. Blackburn, J. van Benthem, and F. Wolter, eds.), Elsevier, Amsterdam, 2006, pp. 249-329.

[18] V. Goranko and D. Vakarelov, Elementary canonical formulae: extending Sahlqvist's theorem, Ann. Pure. Appl. Logic 141 (2006), 180-217.

[19] E. Grädel, The decidability of guarded fixed point logic, JFAK. Essays Dedicated to Johan van Benthem on the Occasion of his 50th Birthday (J. Gerbrandy, M. Marx, M. de Rijke, and Y. Venema, eds.), Vossiuspers, Amsterdam University Press, Amsterdam, 1999, CD-ROM, ISBN 905629104 1 .

[20] M. Kracht, How completeness and correspondence theory got married, Diamonds and Defaults (M. de Rijke, ed.), Kluwer Academic Publishers, 1993, pp. $175-214$.

[21] A. Nonnengart and A. Szałas, A fixpoint approach to second-order quantifier elimination with applications to correspondence theory, Logic at Work: Essays Dedicated to the Memory of Helena Rasiowa (E. Orłowska, ed.), Studies in Fuzziness and Soft Computing, vol. 24, Physica-Verlag, 1999, pp. $307-328$.

[22] H. Sahlqvist, Completeness and correspondence in the first and second order semantics for modal logic, Proc. 3rd Scandinavian logic symposium, Uppsala, 1973 (Amsterdam) (S. Kanger, ed.), North Holland, 1975, pp. 110143.

[23] G. Sambin and V. Vaccaro, A new proof of Sahlqvist's theorem on modal definability and completeness, J. Symbolic Logic 54 (1989), 992-999.

[24] A. Tarski, A lattice-theoretical fixpoint theorem and its applications, Pacific Journal of Mathematics 5 (1955), 285-309.

Johan van Benthem

Institute for Logic, Language \& Computation (ILLC), University of Amsterdam, P.O. Box 94242, 1090 GE Amsterdam, The Netherlands

J.vanBenthem@uva.nl

Nick Bezhanishvili

Department of Computing, Imperial College London, London SW7 2AZ, UK

nbezhani@doc.ic.ac.uk

Ian Hodkinson

Department of Computing, Imperial College London, London SW7 2AZ, UK

i.hodkinson@imperial.ac.uk 